\title{
Analysis of the permeation behavior of ethanol/water mixtures through a polydimethylsiloxane (PDMS) membrane in pervaporation and vapor permeation conditions
}

Hietaharju Jenna, Kangas Jani, Tanskanen Juha

Chemical Process Engineering, University of Oulu, P.O. Box 4300, FI-90014 University of Oulu. Corresponding author: jenna.hietaharju@oulu.fi

\section{Highlights:}

- Mutual interactions between penetrants have importance in the permeation behavior

- Mutual slowing down of penetrants observed

- Permeation behavior analyzed with a Maxwell-Stefan model

- Ethanol-water separations both in pervaporation and vapor permeation conditions

Keywords:

Pervaporation, Vapor permeation, Maxwell-Stefan theory, PDMS membrane, Mass transfer

\begin{abstract}
In this study the interactions between ethanol and water penetrating in a supported polydimethylsiloxane membrane were investigated both using experimental and mathematical modelling work. To reach comprehensive analysis of the membrane performance and the penetrant interactions, the experimental work included a wide range of pervaporation and vapor permeation experiments with pure and binary ethanol-water mixtures. A Maxwell-Stefan model was used to further analyze the membrane performance with a Flory-Huggins formulation to describe the sorption behavior. According to the formed model, both water and ethanol permeation are slowed down from the permeation rates observed with pure feeds and in binary permeation especially the experimental ethanol flux is lower than the flux expected by the model. This suggests that the interactions between the penetrants in PDMS are high resulting to decreased diffusion rate of both ethanol and water.
\end{abstract}




\section{Introduction}

Polymeric membranes, such as polydimethylsiloxane (PDMS) membranes, have received considerable attention in the literature due to their hydrophobicity, and hence their capability to separate organics from dilute aqueous mixtures [1-3], and even to break an azeotrope. Therefore, applying pervaporation or vapor permeation with conventional separation processes can help to reduce operating costs while these hybrid processes are also typically more technologically and economically feasible than a stand-alone membrane process. New pervaporation-distillation or vapor permeation-distillation hybrid processes are constantly suggested to improve the energy efficiency of ethanol recovery or other organics separation tasks. [4-7]. Membranes are also often coupled with bioreactors. Especially, the application of different polymeric membranes to recover bioalcohols, including ethanol, in situ from fermentation broths using pervaporation has attracted considerable attention [8-14].

Currently, PDMS is a favored choice over other hydrophobic polymeric membranes for the separation of dilute ethanol aqueous solutions due to its relatively good separation capabilities and the stability of PDMS in comparison to other polymeric membranes $[9,20]$. The mechanical strength of PDMS membranes can be improved with ceramic support layers [21,22]. The drawback of support layers is the resistance they create for permeation that can have a significant effect on pervaporation performance [20,23-25]. In the case of alcohol separation from aqueous solutions with polymeric membranes, many of the literature studies focus on the study of the separation of dilute alcohol solutions $(<5 \mathrm{wt} . \%)$ at low temperatures by pervaporation $[14,26]$. This is natural as the operating conditions are generally in this range in the applications of product recovery from fermentation broth and, the membranes have typically good separation capabilities in these conditions [26-28]. The analysis of the membrane behavior in the applications can be aided using mathematical models.

Furthermore, mathematical models such as Maxwell-Stefan theory, are usable in the prediction of process performance at different conditions. Thus, mathematical models can be used in the design of a membrane-based separation processes and as a help to reveal the phenomena behind the experimental observations with polymeric membranes $[15,16,27,29]$. The performance of a membrane is often strongly affected by the process conditions, such as changes in the feed concentration, temperature, and pressure on the feed and permeate sides of the membrane that form the driving force for the separation. Thus, in-depth analysis of the phenomena and modeling requires reliable experimental data covering a wide range of temperatures and compositions.

The permeation of the compounds to be separated is dependent on their sorption/desorption and diffusion behavior. The mass transport trough polymeric layers is generally described with the solution-diffusion theories which can be modified to consider the polymer swelling caused by the penetrants [30]. The degree of swelling depends on the molecular size and concentration of the components in the feed solution and is different in the vapor and liquid phases [25,31]. In general, the higher the ethanol concentration in the feed, the higher the swelling [25]. The swelling of the polymer layer can be suppressed by using a rigid ceramic support, that improves the membrane stability [22]. In addition to the penetrant-polymer interactions, also the penetrant-penetrant interactions control the permeation. The interactions between the penetrants are high in ethanol-water solutions and may result in the formation of clusters by hydrogen bonding within the membrane matrix [16,27,32]. This may even decrease the diffusivities of both components simultaneously resulting in mutual-slowing down of the components, not only the faster diffusing component as expected [27,32], thus affecting the achievable separation of a mixture. 
Among the models used to describe the membrane -assisted separation, Maxwell-Stefan theory has been shown practical to describe and predict the diffusion of multicomponent mixtures. The theory relies on irreversible thermodynamics, giving relationship between the driving force and the frictional resistances [29,33,34]. Maxwell-Stefan models have successfully been applied in analysis of pervaporation fluxes to describe multicomponent diffusion through various types of membranes [29,34-38].

In this study, we have performed experiments aiming at providing credible data on both pervaporation and vapor permeation separation of different composition aqueous ethanol mixtures for the analysis of a PDMS membrane characteristics. The data is analyzed with the help of a formed mathematical model using Flory-Huggins formulation for sorption and Maxwell-Stefan equations for permeation. The objective of this study is to evaluate the effect of the solute interactions in the PDMS membrane and yield further evidence on the frictional contributions and mutual-slowing down behavior of the ethanol and water in real PDMS membranes.

\section{Materials and methods}

\subsection{Membrane characteristics}

The composite PDMS membrane was synthesized by the Fraunhofer Institute for Ceramic Technologies and Systems (Germany). The PDMS membrane was coated on the inner surface of a tubular $\alpha-\mathrm{Al}_{2} \mathrm{O}_{3}$ support. The support consisted of four layers, each having specific characteristics in sense of porosity and thickness. The total length of the tubular membrane was $250 \mathrm{~mm}$ and the outer and inner diameters were $10 \mathrm{~mm}$ and $7 \mathrm{~mm}$, respectively. Membrane thickness was $80 \mu \mathrm{m}$. The estimated active surface area of the PDMS membrane was $0.005 \mathrm{~m}^{2}$.

The PDMS composite membrane was imaged with a scanning electron microscope (Center of Microscopy and Nanotechnology, University of Oulu) after the pervaporation and vapor permeation experiments, and based on the imaging, the selective PDMS membrane layer was observed to have sustained its main structural characteristics. In addition, the separation characteristics, i.e. component fluxes and separation factors of the membrane were observed similar to those of a fresh membrane.

\subsection{Analysis methods}

The ethanol concentration of the feed and permeate samples were analyzed with chromatography and density analysis methods. Small permeate samples were analyzed with a gas chromatograph (Agilent Technologies 6890N Network GC) using a thermal conductivity detector (TCD) or by high performance liquid chromatography (Agilent 1200 series HPLC). The gas chromatograph was equipped with a DB-ALC1 column that is suitable for alcohol analysis. An ICSep ICE-Coregel 87H3 (Transgenomic) column was applied in high performance liquid chromatography. External calibration was used in the chromatographic analyses and when necessary, samples were diluted appropriately. Additionally, the ethanol concentrations of the feed and retentate were measured with a density analyzer (Anton Paar DSA 5000).

\subsection{Experimental set-up and conditions}

The separation of the ethanol/water solution was carried out with the set-up illustrated in Fig. 1. The main equipment in the set-up consisted of a feed tank, vaporizer, membrane cell placed inside a thermostatic oven, and permeate collection in cold traps. Both pervaporation and vapor permeation experiments were conducted with the same apparatus but the vaporizer (shown in 
red in Fig. 1) was bypassed when used in the pervaporation mode (illustrated by the thick blue line in Fig. 1).

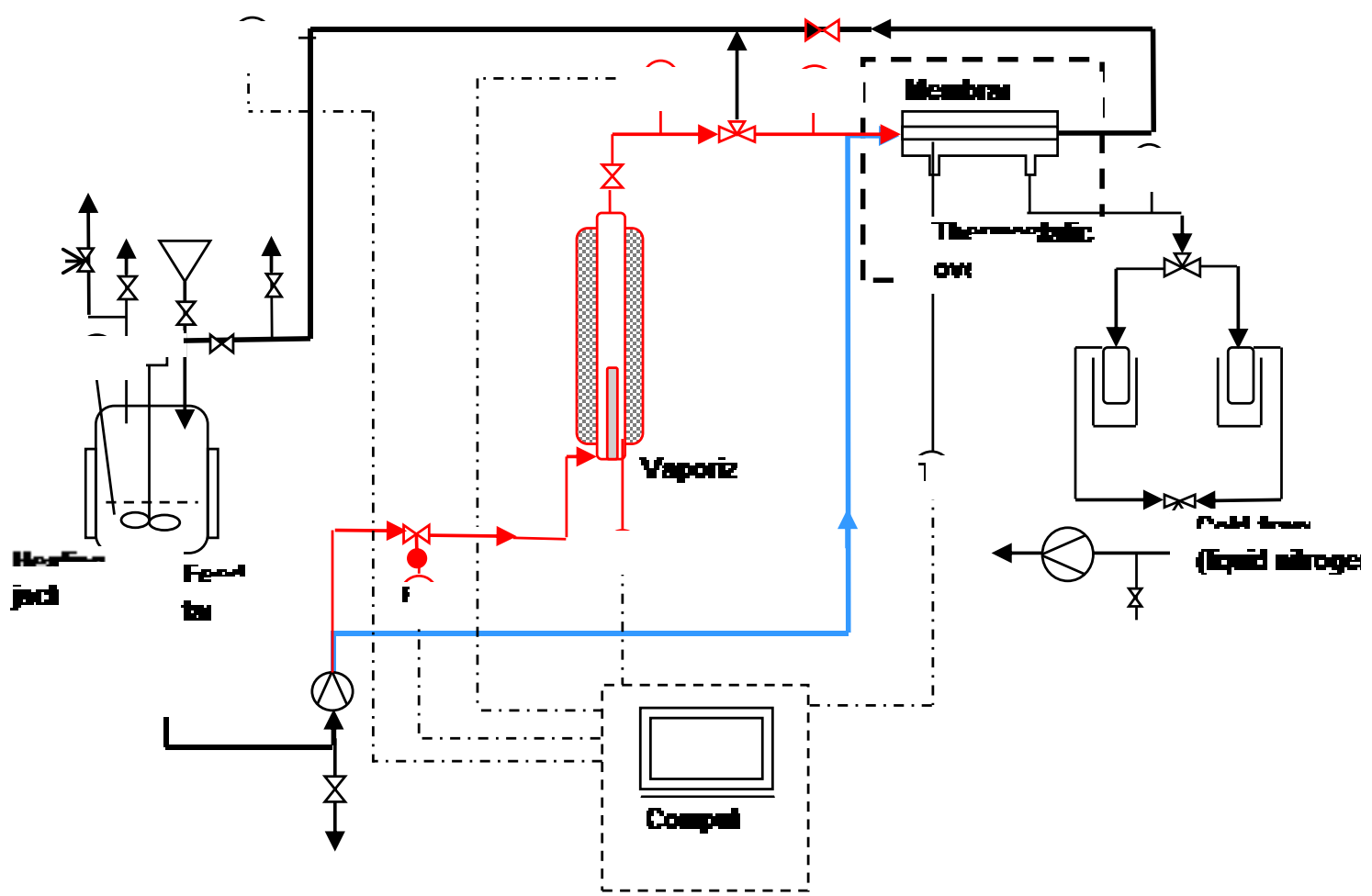

Figure 1. Experimental set-up used in the membrane separation studies. Instruments used exclusively for vapor permeation are shown in red, and pervaporation line is displayed as blue, respectively. The pervaporation experiments were performed using a feeding line with a wider inner diameter, whereas the vapor permeation experiments also included a flow measurement and control device, a heater to vaporize the feed, and a back-pressure valve in the retentate line.

The feed solutions were made by mixing ultrapure water (Milli-Q) with ethanol (absolute, VWR) in selected proportions. The feed volume was considerably larger ( $>2.5$ liter) in comparison to the permeate volume during an experiment. Thus, the change in feed concentration during an experiment can be considered constant. The feed flow rate was measured to be approx. $5.5 \mathrm{l} / \mathrm{min}$ that is sufficient to maintain the flow in a turbulent region in all experimental conditions (Reynolds number was 9600-25300 at $40{ }^{\circ} \mathrm{C}$ ). In the vapor permeation experiments, a mass flow meter and controller (mini Cori-Flow, Bronkhorst) was used to measure and control the flow on-line. The flow rate was maintained at $100 \mathrm{~g} / \mathrm{min}$ in all the vapor permeation experiments. At this flow rate, the vapor flow is turbulent at the feed side of the membrane in the experimental conditions $(\mathrm{Re}=22000-27600)$. Thus, it can be concluded that the feed flow is well mixed in the cross-sectional direction of the feed flow both in pervaporation and vapor permeation conditions. The turbulent flow region diminishes the effect of the feed flow rate on total flux and separation performance [27]. Therefore, concentration polarization and the effect of the flow rate on process performance were regarded negligible in the experimental condition due to the high Reynolds number.

The experiment temperatures in the majority of the pervaporation experiments were either $40{ }^{\circ} \mathrm{C}$ or $60{ }^{\circ} \mathrm{C}$. A few pervaporation experiments were also performed at the temperatures of 25,70 , and $90{ }^{\circ} \mathrm{C}$ using a constant feed composition to obtain temperature dependence for the 
separation over a wide temperature range. In the vapor permeation experiments, the temperature was held a few degrees above the dew point of the feed solution to ensure the vapor phase feed.

The membrane was placed in a stainless-steel housing inside a thermostatic oven. The housing had connections to the feed, retentate, permeate (vacuum), and a permeate side thermocouple, as presented in Fig. 1. The feed was preheated in the feed tank in the pervaporation experiments, while in the vapor permeation experiments the feed flow was heated and vaporized in the vertical tubular oven (shown in Fig. 1), which was filled with steel rods as packing material. The permeate condensed in cold traps that were held submerged in liquid nitrogen in Dewar flasks. The permeate pressure varied from 1720 to $2140 \mathrm{~Pa}$ in the experiments. There were two parallel condensation loops to enable continuous permeate collection. The number of permeate samples collected and the sample interval in each experiment varied depending on the total flux. A minimum of three steady-state samples were taken in each experiment.

\subsection{Performance Characteristics}

The performance characteristics of a membrane can be analyzed by evaluating the driving force of separation and the observed flux and separation factor. Flux describes the mass transfer rate of compounds through the membrane area. In this study, the steady-state mass flux $(J)$ was determined based on the mass of defrosted permeate sample $(m,[\mathrm{~kg}])$, sampling time $(t,[\mathrm{~h}])$, and membrane area $\left(A,\left[\mathrm{~m}^{2}\right]\right)$.

$$
J=\frac{m}{A t}
$$

The separation factor $\left(\alpha_{i / j}\right)$ for the separation of component $i$ from component $j$ was determined as the ratio of the ratios of component mass fractions, or correspondingly mole fractions, in the permeate and feed:

$$
\alpha_{i / j}=\frac{\left(w_{i} / w_{j}\right)_{\text {perm }}}{\left(w_{i} / w_{j}\right)_{\text {feed }}},
$$

where $w_{i}$ and $w_{j}$ are the mass fractions of compound $i$ and $j$ either on the permeate (perm) or feed side (feed), respectively.

Baker et al. [39] recommend the use of selectivity and permeances instead of flux and separation factor in the evaluation of the membrane performance, and its intrinsic properties. Thus, to enable easier comparison between the performances of different membranes, the membrane selectivity coefficient $\sigma_{i / j}$ was also evaluated:

$$
\sigma_{i / j}=\Pi_{i} / \Pi_{j},
$$

where the permeance of component $i, \Pi_{i}$ is defined as

$$
\Pi_{i}=\frac{J_{i}}{\Delta f_{i}}
$$

where $J_{i}$ is the molar flux of component $i$, and $\Delta f_{i}$ the corresponding driving force.

In general, the driving force for pervaporation is given by the chemical potential gradients of each compound across the membrane. As a result, the driving force can be evaluated based on the difference between the feed and permeate side fugacities of component $i, f_{i \text {,feed }}[\mathrm{Pa}]$ and $f_{i, \text { perm }}[\mathrm{Pa}]$ :

$$
\Delta f_{i}=f_{i, \text { feed }}-f_{i, \text { perm }}
$$


where the fugacities in the feed $f_{i, \text { feed }}$ can be described with an activity coefficient model that takes into account the non-idealities of the ethanol-water liquid feed

$$
f_{i, \text { feed }}=x_{i} \gamma_{i} P_{i}^{\mathrm{sat}}
$$

and the permeate is assumed to behave as an ideal gas mixture due to the low pressure, and permeate side fugacity $f_{i \text {,perm }}$ is described with

$$
f_{i, \mathrm{perm}}=y_{i} P_{\mathrm{perm}}
$$

where $x_{i}$ is the mole fraction of component $i$ in the liquid feed mixture, $\gamma_{i}$ is the activity coefficient of component $i$ in the liquid feed mixture, and $P_{i}^{\text {sat }}$ is the vapor pressure of component $i[\mathrm{~Pa}], y_{i}$ is the mole fraction of component $i$ in the vapor phase on the permeate side and $P_{\text {perm }}$ is the permeate pressure $[\mathrm{Pa}]$.

The feed side fugacities for ethanol and water were evaluated in the Aspen Plus V8.8 simulation environment using the UNIQ-HOC thermodynamic model. Due to the interactions between ethanol and water in the liquid feed, the feed side fugacities depend not only on the temperature, but also on the mole fractions of ethanol and water in the mixture. In a non-ideal liquid mixture, the differences in the values of activity coefficients $\gamma_{i}$, and the vapor pressures of components affect significantly on the observed separation factor. In contrast, for pressures near atmospheric pressure and below, as used in the experiments, the vapor phase ethanol/water mixture can be considered as an ideal gas mixture. Consequently, the fugacity of a component on the feed side can be calculated analogously to Eq. (7) with $f_{i, \text { feed }}=y_{i, \text { feed }} P_{\text {feed }}$, where $y_{i, \text { feed }}$ is the mole fraction of component $i$ in the vapor phase on the feed side of the membrane.

The measured permeate concentration $y_{i}$ and pressure $P_{\text {perm }}$ may differ from the actual concentration and pressure at the downstream interface of the PDMS because of the resistance caused by the support layers. In addition to the sorption and diffusion behavior in the PDMS layer, the support may create a significant contribution to the total mass transfer resistance over a membrane [20,23,40,41], and this should be considered in calculation of the driving force. The PDMS membrane used in this study is laid over a ceramic support that has four layers with different porosities. The flux trough the porous support is estimated using Dusty Gas Model [40,41], taking into account Knudsen diffusion and Poiseuille flow that is described in more detail in supplementary material. The mass transport depends on the pore size and tortuosity as well as the thickness of the porous layer. The pore sizes and thicknesses of the support layers were obtained from the Fraunhofer Institute for Ceramic Technologies and Systems (Germany). Tortuosity was estimated using the values from Weyd et al. [40]. The physical properties for the permeate flow for each temperature and ethanol concentration case were obtained with Aspen Plus V8.8 using the UNIQ-HOC property method.

The support contribution to the total mass transfer resistance was calculated to be negligible regarding the permeation of ethanol-water mixture through the PDMS/ceramic composite membrane in all experimental conditions. The total fugacity difference, or the pressure drop, across the support layers was approximated to make up only $0.5 \%$ of the total fugacity difference over the composite membrane including both the PDMS membrane and the support. Therefore, the reported values that are based on the permeate side conditions can be considered very good approximations of the separation efficiency of the PDMS membrane layer itself. In this case, the PDMS layer is relatively thick $(80 \mu \mathrm{m})$, which means naturally that the observed fluxes are relatively small, not creating large pressure drop. However, for high-flux thin membrane materials, the support effect to the flux and the membrane selectivity should be considered. 


\subsection{Modelling permeation}

\subsubsection{Sorption}

Sorption of ethanol and water on the feed side and the permeate side of the PDMS layer were described with an extended Flory-Huggins model. The extended Flory-Huggins model can be used to calculate the activities of the penetrants, $a_{1}$ and $a_{2}$, in the PDMS, when the interaction parameters between the penetrants and the polymeric membrane are dependent on the volume fractions of the compounds permeating through the membrane [29]:

$$
\begin{gathered}
\ln a_{1, \mathrm{~m}}=\ln \varphi_{1}+\left(1-\varphi_{1}\right)-\varphi_{2} \frac{\bar{V}_{1}}{\bar{V}_{2}}-\varphi_{\mathrm{m}} \frac{\bar{V}_{1}}{\bar{V}_{\mathrm{m}}}+\left(\chi_{12} \varphi_{2}+\chi_{1 \mathrm{~m}} \varphi_{\mathrm{m}}\right)\left(\varphi_{2}+\varphi_{\mathrm{m}}\right) \\
-\chi_{2 \mathrm{~m}} \frac{\bar{V}_{1}}{\bar{V}_{2}} \varphi_{2} \varphi_{\mathrm{m}}-u_{1} u_{2} \varphi_{2} \frac{\partial \chi_{12}}{\partial u_{2}}-u_{1} u_{2} \varphi_{\mathrm{m}} \frac{\partial \chi_{1 \mathrm{~m}}}{\partial u_{2}}-\varphi_{1} \varphi_{\mathrm{m}}^{2} \frac{\partial \chi_{1 \mathrm{~m}}}{\partial \varphi_{\mathrm{m}}} \\
+\frac{\bar{V}_{1}}{\bar{V}_{2}} u_{2}^{2} \varphi_{\mathrm{m}} \frac{\partial \chi_{2 \mathrm{~m}}}{\partial u_{1}}-\frac{\bar{V}_{1}}{\bar{V}_{2}} \varphi_{2} \varphi_{\mathrm{m}}^{2} \frac{\partial \chi_{2 \mathrm{~m}}}{\partial \varphi_{\mathrm{m}}} \\
\ln a_{2, \mathrm{~m}}=\ln \varphi_{2}+\left(1-\varphi_{2}\right)-\varphi_{1} \frac{\bar{V}_{2}}{\bar{V}_{1}}-\varphi_{\mathrm{m}} \frac{\bar{V}_{2}}{\bar{V}_{\mathrm{m}}}+\left(\chi_{12} \varphi_{1} \frac{\bar{V}_{2}}{\bar{V}_{1}}+\chi_{2 \mathrm{~m}} \varphi_{\mathrm{m}}\right)\left(\varphi_{1}+\varphi_{\mathrm{m}}\right) \\
-\chi_{1 \mathrm{~m}} \frac{\bar{V}_{2}}{\bar{V}_{1}} \varphi_{1} \varphi_{\mathrm{m}}+\frac{\bar{V}_{2}}{\bar{V}_{1}} u_{1}^{2} \varphi_{2} \frac{\partial \chi_{12}}{\partial u_{2}}+\frac{\bar{V}_{2}}{\bar{V}_{1}} u_{1}^{2} \varphi_{\mathrm{m}} \frac{\partial \chi_{1 \mathrm{~m}}}{\partial u_{2}}-\frac{\bar{V}_{2}}{\bar{V}_{1}} \varphi_{1} \varphi_{\mathrm{m}}^{2} \frac{\partial \chi_{1 \mathrm{~m}}}{\partial \varphi_{\mathrm{m}}} \\
-u_{1} u_{2} \varphi_{\mathrm{m}} \frac{\partial \chi_{2 \mathrm{~m}}}{\partial u_{1}}-\varphi_{2} \varphi_{\mathrm{m}}^{2} \frac{\partial \chi_{2 \mathrm{~m}}}{\partial \varphi_{\mathrm{m}}}
\end{gathered}
$$

where $\varphi_{i}$ is the volume fraction of compound $i=1,2$ (ethanol, water), $\varphi_{\mathrm{m}}$ is the volume fraction of the polymer, $\bar{V}_{i}$ is the partial molar volume of compound $i=1,2, \mathrm{~m}, \chi_{12}$ is the interaction parameter between the permeating compounds 1 and $2, \chi_{1 \mathrm{~m}}$ is the interaction parameter between compound 1 and the polymer, $\chi_{2 \mathrm{~m}}$ is the interaction parameter between compound 2 and the polymer and $u_{i}=\frac{\varphi_{i}}{\varphi_{1}+\varphi_{2}}, i=1,2$. It was assumed that the penetrant-polymer interactions $\chi_{i \mathrm{~m}}$ can be presented using the form[42] :

$$
\chi_{i \mathrm{~m}}=a_{i \mathrm{~m}}(T)+\frac{b_{i \mathrm{~m}}(T)}{\left(1+c_{i \mathrm{~m}}(T) \varphi_{\mathrm{m}}\right)^{2}}
$$

where $a_{i \mathrm{~m}}(T), b_{i \mathrm{~m}}(T)$ and $c_{i \mathrm{~m}}(T)$ are temperature dependent penetrant $i$ polymer interaction parameters. Due to the form of Eq. (8),

$$
\frac{\partial \chi_{i \mathrm{~m}}}{\partial \varphi_{\mathrm{m}}}=\frac{-2 b_{i \mathrm{~m}}(T) c_{i \mathrm{~m}}(T)}{\left(1+c_{i \mathrm{~m}}(T) \varphi_{\mathrm{m}}\right)^{3}} ; \frac{\partial \chi_{1 \mathrm{~m}}}{\partial u_{2}}=0 ; \frac{\partial \chi_{2 \mathrm{~m}}}{\partial u_{1}}=0 .
$$

The temperature dependence of $\chi_{1 \mathrm{~m}}$ was selected as:

$$
\begin{aligned}
& a_{1 \mathrm{~m}}(T)=a_{1 \mathrm{~m}, \mathrm{ref}}+a_{1 \mathrm{~m}, 1}\left(T-T_{\mathrm{ref}}\right) ; \\
& b_{1 \mathrm{~m}}(T)=b_{1 \mathrm{~m}, \mathrm{ref}}+b_{1 \mathrm{~m}, 1}\left(1-T_{\mathrm{ref}} / T\right) ; \\
& c_{1 \mathrm{~m}}(T)=c_{1 \mathrm{~m}, \mathrm{ref}}+c_{1 \mathrm{~m}, 1}\left(1-T_{\mathrm{ref}} / T\right),
\end{aligned}
$$

where $a_{1 \mathrm{~m}, \mathrm{ref}}, b_{1 \mathrm{~m}, \mathrm{ref}}$ and $c_{1 \mathrm{~m} \text {,ref }}$ are the interaction parameters between penetrant 1 and the polymer at the reference temperature, $a_{1 \mathrm{~m}, 1}, b_{1 \mathrm{~m}, 1}$ and $c_{1 \mathrm{~m}, 1}$ are the temperature dependence parameters between penetrant 1 and the polymer, $T_{\text {ref }}$ is the used reference temperature, $298.15 \mathrm{~K}$. The form of the temperature dependence was selected based on the evaluation of different alternatives with respect to the available experimental data. Further information of this 
can be found from the supplementary data file. Correspondingly, the temperature dependence of $\chi_{2 \mathrm{~m}}$ was selected as:

$$
\begin{aligned}
& a_{2 \mathrm{~m}}(T)=a_{2 \mathrm{~m}, \mathrm{ref}}+a_{2 \mathrm{~m}, 1}\left(1-T_{\mathrm{ref}} / T\right) ; \\
& b_{2 \mathrm{~m}}(T)=b_{2 \mathrm{~m}, \mathrm{ref}}+b_{2 \mathrm{~m}, 1}\left(1-T_{\mathrm{ref}} / T\right) \\
& c_{2 \mathrm{~m}}(T)=c_{2 \mathrm{~m}, \mathrm{ref}}+c_{2 \mathrm{~m}, 1}\left(1-T_{\mathrm{ref}} / T\right) .
\end{aligned}
$$

The extended Flory-Huggins model can be applied also to describe the binary bulk liquid phase activities, i.e. when the polymer is not present in the solution, $\varphi_{\mathrm{m}}=0$. In this case the equations are simplified in the form $\left(\varphi_{1}+\varphi_{2}=1\right)$ :

$$
\begin{aligned}
& \ln a_{1}=\ln \varphi_{1}+\left(1-\varphi_{1}\right)-\varphi_{2} \frac{\bar{V}_{1}}{\bar{V}_{2}}+\left(\chi_{12} \varphi_{2}^{2}\right)-\varphi_{1} \varphi_{2}^{2} \frac{\partial \chi_{12}}{\partial \varphi_{2}} \\
& \ln a_{2}=\ln \varphi_{2}+\left(1-\varphi_{2}\right)-\varphi_{1} \frac{\bar{V}_{2}}{\bar{V}_{1}}+\left(\chi_{12} \varphi_{1}^{2} \frac{\bar{V}_{2}}{\bar{V}_{1}}\right)+\frac{\bar{V}_{2}}{\bar{V}_{1}} \varphi_{1}^{2} \varphi_{2} \frac{\partial \chi_{12}}{\partial \varphi_{2}}
\end{aligned}
$$

where $\chi_{12}$ is the penetrant 1 - penetrant 2 interaction parameter. $\chi_{12}$ has the following dependence of $u_{2}$ at constant temperature:

$$
\chi_{12}\left(u_{2}, T\right)=a_{12}(T)+b_{12}(T) u_{2}+c_{12}(T) u_{2}^{2}+d_{12}(T) u_{2}^{3}+e_{12}(T) u_{2}^{4},
$$

where $a_{12}(T), b_{12}(T), c_{12}(T), d_{12}(T)$, and $e_{12}(T)$ are temperature dependent interaction parameters between penetrants 1 and 2 . The temperature dependency of $\chi_{12}$ is introduced by giving a temperature dependency for each parameter in Eq. (16). Due to the form of Eq. (16) at constant temperature,

$$
\left(\frac{\partial \chi_{12}}{\partial \varphi_{2}}\right)_{T}=\left(\frac{\partial \chi_{12}}{\partial u_{2}}\right)_{T}=b_{12}(T)+2 c_{12}(T) u_{2}+3 d_{12}(T) u_{2}^{2}+4 e_{12}(T) u_{2}^{3}
$$

The data for evaluating the parameters in Eq. (14) was obtained using the WILS-HOC databank binary interaction parameters for ethanol and water available at Aspen Plus (v8.8). The forms of the resulting temperature dependences were:

$$
\begin{aligned}
& a_{12}(T)=a_{12, \text { ref }}-a_{12,1}\left(\frac{1}{T_{\text {ref }}}-\frac{1}{T}\right) ; \\
& b_{12}(T)=b_{12, \text { ref }}-b_{12,1}\left(\frac{1}{T_{\text {ref }}}-\frac{1}{T}\right) ; \\
& c_{12}(T)=c_{12, \text { ref }}-c_{12,1}\left(\frac{1}{T_{\text {ref }}}-\frac{1}{T}\right) ; \\
& d_{12}(T)=d_{12, \text { ref }}-d_{12,1}\left(\frac{1}{T_{\text {ref }}}-\frac{1}{T}\right) ; \\
& e_{12}(T)=e_{12, \text { ref }}
\end{aligned}
$$

where $a_{12 \text {,ref }}, b_{12 \text {,ref }}, c_{12 \text {,ref }}, d_{12 \text {,ref }}$ and $e_{12 \text {,ref }}$ are penetrant $1-2$ interaction parameters at the reference temperature, and $a_{12,1}, b_{12,1}, c_{12,1}, d_{12,1}$ and $e_{12,1}$ are penetrant $1-2$ interaction temperature dependence parameters. Thus, as a summary of Eqs. (18)-(22), all the other parameters have a temperature dependence, except $e_{12}$. This selection was based on the performed parameter estimation results evaluation. The parameter values for the temperature dependence of $\chi_{12}$ and fit to the data from Aspen Plus are presented in the supplementary data file. 
Both on the feed-PDMS and PDMS-permeate interfaces the sorption equilibrium is assumed. Thus, the chemical potential, and activity, of a compound in the bulk fluid phase and on the surface of the PDMS membrane is equal:

$$
\ln a_{i, \mathrm{bulk}}=\ln a_{i, \mathrm{~m}}
$$

where $a_{i, \text { bulk }}$ is the activity of compound $i$ in the bulk fluid phase and $a_{i, \mathrm{~m}}$ is the activity of compound $i$ on the surface of PDMS. If the bulk fluid phase is vapor, the activity of a compound in the bulk fluid is evaluated using the expression:

$$
\ln a_{i, \mathrm{bulk}}=\ln \left(\frac{f_{i, \mathrm{bulk}}}{f_{i}^{\text {sat }}}\right)=\ln \left(\frac{y_{i} P}{P_{i}^{\text {sat }}}\right) .
$$

Instead, if the bulk fluid phase is liquid, the activity of ethanol and water in the bulk fluid are evaluated using Eqs. (14) and (15), respectively.

\subsubsection{Diffusion and pervaporative flux}

A common way to describe the permeation through a polymeric film is the solution-diffusion model using Fick's law:

$$
J_{i}=-D_{i, \mathrm{~m}} \frac{\mathrm{d} C_{i}}{\mathrm{~d} z}
$$

where $D_{i, \mathrm{~m}}$ is the specific diffusion coefficient of component $i$ for the diffusion in a polymeric membrane $\left[\mathrm{m}^{2} \mathrm{~s}^{-1}\right], C_{i}$ is the concentration of component $i$ in the membrane at the depth of $z[\mathrm{~kg}$ $\left.\mathrm{m}^{-3}\right], J_{i}$ is the component flux through the membrane $\left[\mathrm{kg} \mathrm{m}^{-2} \mathrm{~s}^{-1}\right]$ and $z$ denotes the depth of the membrane $[\mathrm{m}]$. The diffusion coefficient of a single compound permeating through a polymeric membrane can generally be expressed as [43]

$$
D_{i, \mathrm{~m}}=D_{i 0} \exp \left(\varepsilon_{i i} w_{i}\right)
$$

where $D_{i 0}$ is the diffusion coefficient of component $i$ at infinite dilution, $w_{i}$ is the mass fraction of the component in the membrane, and $\varepsilon_{i i}$ is the plasticization coefficient between components $i$ and $i$ in the polymeric membrane matrix. Correspondingly, the diffusion coefficients $D_{i, \mathrm{~m}}$ $(i=1,2)$ in binary mixture diffusion in the polymeric matrix can generally be expressed as [30]

$$
D_{i, \mathrm{~m}}=D_{i 0} \exp \left(\varepsilon_{i i} w_{i}+\varepsilon_{i j} w_{j}\right)
$$

where $\varepsilon_{i j}$ is the plasticization coefficient between components $i$ and $j$ in the membrane. As both the diffusion coefficients and thus also the fluxes of the permeating components are interdependent, solving the solution-diffusion model in binary mixture permeation demands simultaneous solving of Eq. (25) for both penetrants. The interactions between two penetrants permeating through the membrane can be taken into account with a Maxwell-Stefan model [36]:

$$
\begin{aligned}
& J_{1}=D_{1, \mathrm{~m}}\left(\frac{w_{1} D_{2, \mathrm{~m}}+D_{12}}{D_{12}+w_{1} D_{2, \mathrm{~m}}+w_{2} D_{1, \mathrm{~m}}}\right) \rho_{\mathrm{m}} \frac{d w_{1}}{d z}+D_{1, \mathrm{~m}}\left(\frac{w_{1} D_{2, \mathrm{~m}}}{D_{12}+w_{1} D_{2, \mathrm{~m}}+w_{2} D_{1, \mathrm{~m}}}\right) \rho_{\mathrm{m}} \frac{d w_{2}}{d z} \\
& J_{2}=D_{2, \mathrm{~m}}\left(\frac{w_{2} D_{1, \mathrm{~m}}+D_{12}}{D_{12}+w_{2} D_{1, \mathrm{~m}}+w_{1} D_{2, \mathrm{~m}}}\right) \rho_{\mathrm{m}} \frac{d w_{2}}{d z}+D_{2, \mathrm{~m}}\left(\frac{w_{2} D_{1, \mathrm{~m}}}{D_{12}+w_{2} D_{1, \mathrm{~m}}+w_{1} D_{2, \mathrm{~m}}}\right) \rho_{\mathrm{m}} \frac{d w_{1}}{d z}
\end{aligned}
$$

where $D_{12}$ is the binary diffusion coefficient between the permeating components, $D_{i, \mathrm{~m}}$ is the binary diffusion coefficient between component $i(i=1,2)$ and the membrane $\mathrm{m}, \rho_{\mathrm{m}}\left[\mathrm{kg} \mathrm{m}^{-3}\right]$ is the membrane density. Large value of $D_{12}$ means independent diffusion of the components 
within the membrane while a value near zero indicates strong coupling effects where a slower molecule slows down the diffusion of the faster molecule.

When the interdependencies between the permeating components are low, the solving may be performed independently for each permeating component. This situation is possible when their concentrations are low, or the swelling caused by the adsorbed penetrants is low. Since PDMS is a hydrophobic material, the sorbed amount of water is small in comparison to the one of ethanol nearly throughout the investigated operating condition range in this study. On the other hand, solving Eqs. (28) and (29) is time consuming. As a result, the approximate forms of Eqs. (28) and (29) can be used:

$$
\begin{aligned}
& J_{1}=\bar{D}_{1, \mathrm{~m}}\left(\frac{w_{1} \bar{D}_{2, \mathrm{~m}}+D_{12}}{D_{12}+w_{1} \bar{D}_{2, \mathrm{~m}}+w_{2} \bar{D}_{1, \mathrm{~m}}}\right) \rho_{\mathrm{m}} \frac{\Delta w_{1}}{\delta}+\bar{D}_{1, \mathrm{~m}}\left(\frac{w_{1} \bar{D}_{2, \mathrm{~m}}}{D_{12}+w_{1} \bar{D}_{2, \mathrm{~m}}+w_{2} \bar{D}_{1, \mathrm{~m}}}\right) \rho_{\mathrm{m}} \frac{\Delta w_{2}}{\delta} \\
& J_{2}=\bar{D}_{2, \mathrm{~m}}\left(\frac{w_{2} \bar{D}_{1, \mathrm{~m}}+D_{12}}{D_{12}+w_{2} \bar{D}_{1, \mathrm{~m}}+w_{1} \bar{D}_{2, \mathrm{~m}}}\right) \rho_{\mathrm{m}} \frac{\Delta w_{2}}{\delta}+\bar{D}_{2, \mathrm{~m}}\left(\frac{w_{2} \bar{D}_{1, \mathrm{~m}}}{D_{12}+w_{2} \bar{D}_{1, \mathrm{~m}}+w_{1} \bar{D}_{2, \mathrm{~m}}}\right) \rho_{\mathrm{m}} \frac{\Delta w_{1}}{\delta}
\end{aligned}
$$

where $\bar{D}_{i, \mathrm{~m}}$ is the average diffusion coefficient through the membrane. The solution of the approximate equations results, according to Heintz \& Stephan [36], in a relatively small error, less than 5\% in comparison to the exact solution and thus Eqs. (30) and (31) are used in this study. The averaged diffusion coefficients for the penetrants in the membrane $\bar{D}_{i, \mathrm{~m}}$ are defined as [36]:

$$
\bar{D}_{i, \mathrm{~m}}=\frac{\int_{w_{i, F}}^{w_{i, P}} D_{i, \mathrm{~m}} d w_{i}}{w_{i, F}-w_{i, P}}
$$

where $w_{i, F}$ and $w_{i, P}$ are the mass fractions of ethanol $(i=1)$ or water $(i=2)$ at the feed and permeate sides of the membrane, respectively. $D_{i, \mathrm{~m}}$ is expressed with Eq. (27) in Eq. (32). The diffusion coefficients $D_{i, \mathrm{~m}}$ of the penetrants are generally interdependent through the plasticization factors $\varepsilon_{i j}$, as formulated in Eq. (27). However, when the water sorption levels are much less than the ethanol uptake, the effect of the water sorption can be neglected in Eq. (27) as was assumed by Yang and Lue [15]. Instead, at high water feed concentrations the water sorption level is at a comparable level to the one of ethanol and should thus not be ignored. We include the effect of the component having smaller concentration sorbed in the membrane by assuming that it remains constant across the membrane. As a result, the concentration range can be divided into two ranges depending on which penetrant has higher concentration than the other in the membrane. For the range where the adsorbed concentration of ethanol is higher than the concentration of water, the average diffusion coefficients for ethanol (1) and water (2) are written as

$$
\begin{aligned}
& \bar{D}_{1, \mathrm{~m}}=\frac{D_{10}\left[\exp \left(\varepsilon_{11} w_{1, F}+\varepsilon_{12} \bar{w}_{2}\right)-\exp \left(\varepsilon_{11} w_{1, P}+\varepsilon_{12} \bar{w}_{2}\right)\right]}{\varepsilon_{11}\left(w_{1, F}-w_{1, P}\right)}, \\
& \bar{D}_{2, \mathrm{~m}}=\frac{D_{10}\left[\exp \left(\varepsilon_{21} w_{1, F}+\varepsilon_{22} \bar{w}_{2}\right)-\exp \left(\varepsilon_{21} w_{1, P}+\varepsilon_{22} \bar{w}_{2}\right)\right]}{\varepsilon_{21}\left(w_{1, F}-w_{1, P}\right)},
\end{aligned}
$$

and the concentration of water is assumed to be constant across the membrane thickness $\bar{w}_{2}=$ $\left(w_{2, F}+w_{2, P}\right) / 2$.

For the range where the adsorbed concentration of water at the feed side exceeds the ethanol concentration, $\bar{D}_{1, \mathrm{~m}}$ and $\bar{D}_{2, \mathrm{~m}}$ are assumed to be functions of the water mass fraction, while the 
ethanol concentration is approximated constant across the membrane by $\bar{w}_{1}=\left(w_{1, F}+\right.$ $\left.w_{1, P}\right) / 2$.

$$
\begin{aligned}
& \bar{D}_{1, \mathrm{~m}}=\frac{D_{10}\left[\exp \left(\varepsilon_{11} \bar{w}_{1}+\varepsilon_{12} w_{2, F}\right)-\exp \left(\varepsilon_{11} \bar{w}_{1}+\varepsilon_{12} w_{2, P}\right)\right]}{\varepsilon_{12}\left(w_{2, F}-w_{2, P}\right)} ; \\
& \bar{D}_{2, \mathrm{~m}}=\frac{D_{20}\left[\exp \left(\varepsilon_{21} \bar{w}_{1}+\varepsilon_{22} w_{2, F}\right)-\exp \left(\varepsilon_{21} \bar{w}_{1}+\varepsilon_{22} w_{2, P}\right)\right]}{\varepsilon_{22}\left(w_{2, F}-w_{2, P}\right)} .
\end{aligned}
$$

This simplification considers the presence of the minor component but still enables analytical integration of Eq. (32). Especially in the range where water uptake exceeds the ethanol uptake, it is theoretically important to consider the effect of both component to the diffusion. The concentration of ethanol is more controlling factor in diffusion of both components as the plasticization effect of ethanol is higher [15].

\section{Results and discussion}

\subsection{Pervaporation and vapor permeation experiments}

The effects of changing the feed concentrations and temperature were investigated with the pervaporation (PV) and vapor permeation (VP) experiments shown in Fig. 2. As a summary of the experimental results, it can be stated that the ethanol flux increases with increasing ethanol feed mass fraction and temperature. For water the trends are not as clear as for ethanol. The increase of the temperature increases the water flux, while at constant temperature the increase of water feed concentration does not necessarily result in the increase of water flux. 

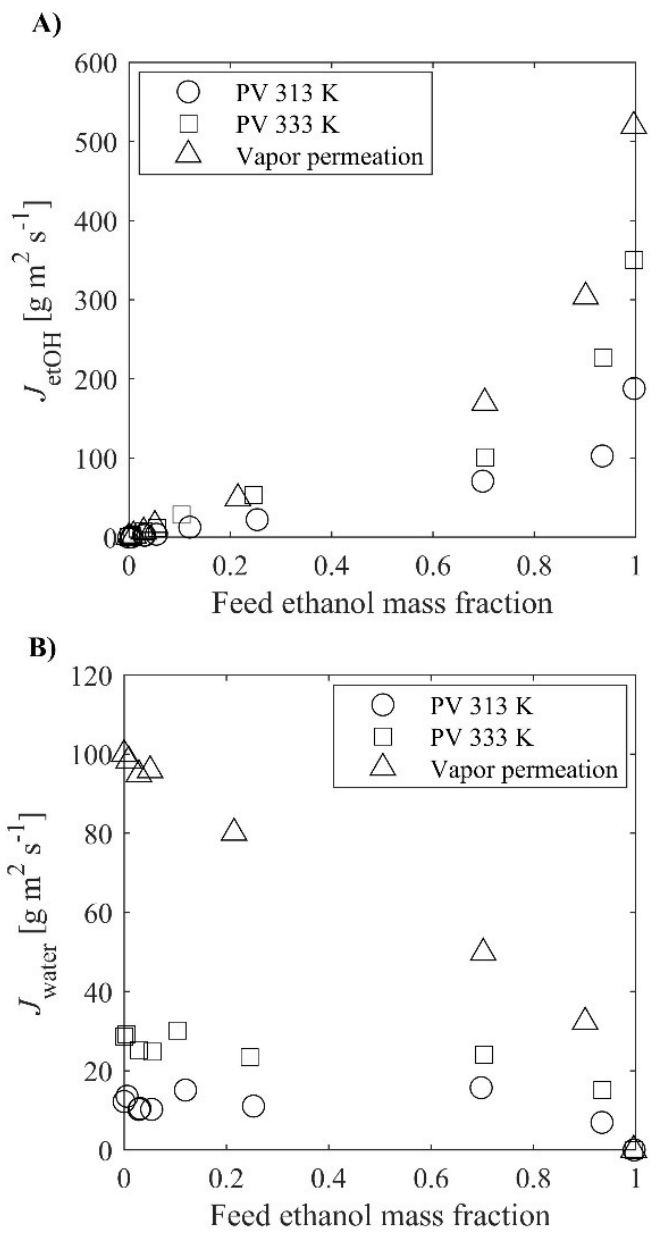

Figure 2. Experimental fluxes of a) ethanol and b) water at different temperatures.

The behavior seen in Fig. 2 is in correlation with the component specific fugacity gradients. Ethanol is more volatile in the experimented conditions, and its fugacity, as expressed in Eq. (6), increases more rapidly as the vapor pressure increases with temperature. On the other hand, the activity coefficient of ethanol $\gamma_{E}$ is higher and makes it more fugitive than water in the experimental conditions. The correlation of the molar flux of both ethanol and water with their corresponding fugacity gradients across the PDMS layer are presented in Fig. 3 in terms of their normalized fluxes and fugacity gradients. The normalization was done independently for each temperature set in pervaporation $(313 \mathrm{~K}$ and $333 \mathrm{~K})$, and for vapor permeation comparing the component specific flux and fugacity gradient at different concentrations to those of the pure component at given temperature and phase. In vapor permeation experiments the temperature changes according to the vapor pressure of the mixture, ensuring one phase conditions, but is treated similarly to the isotherms of pervaporation experiments. 

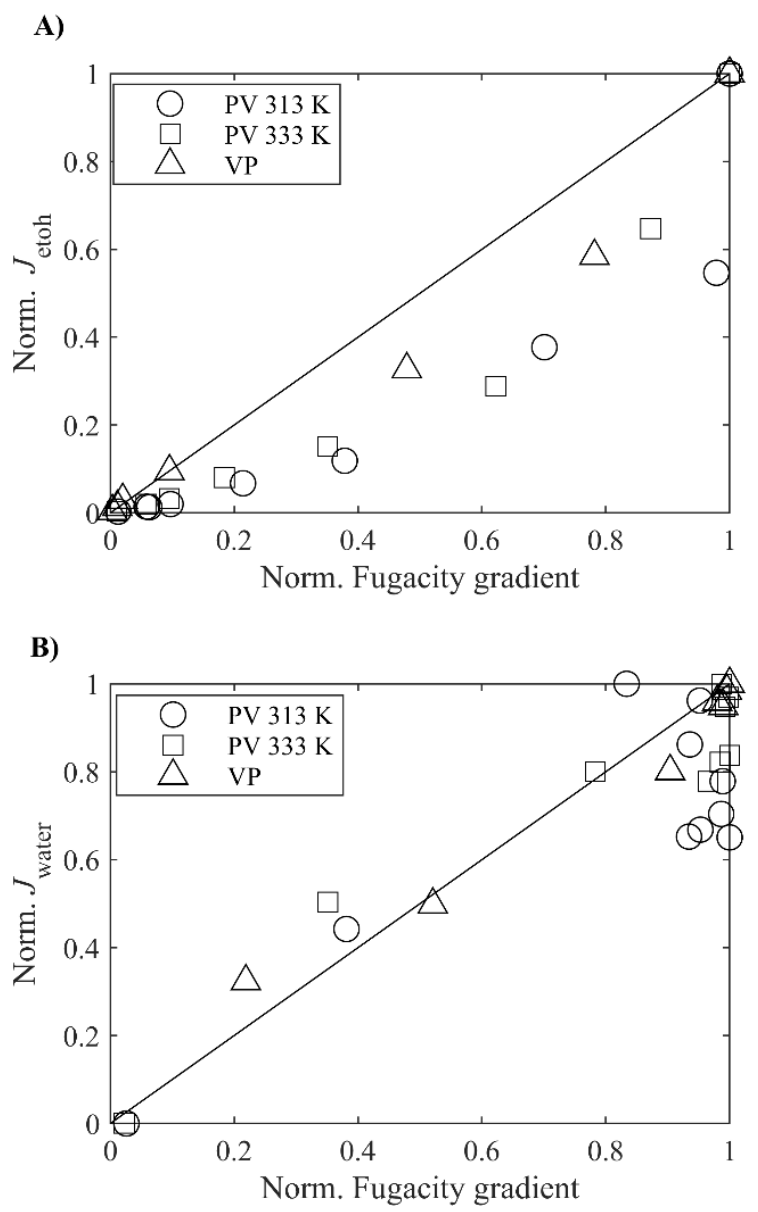

Figure 3. Normalized molar flux of a) ethanol and b) water as a function of normalized fugacity gradient at different temperatures and feed compositions. The normalization is done for each set by dividing the component-specific flux or fugacity gradient at different concentrations by the corresponding flux or fugacity gradient of pure component at the given temperature $(313 \mathrm{~K}$ or $333 \mathrm{~K}$ ) or in vapor phase conditions. Solid line emphasizes $x=y$.

As can be seen in Fig. 3a, the ethanol flux increases as a function of the fugacity gradient of ethanol across the PDMS layer at constant temperature. For water the trend is more mixed at high fugacity gradient range as shown in Fig. 3b. This in turn corresponds majorly the conditions where ethanol feed concentration is low. This implies that especially at these conditions there are significant interactions between the penetrants through sorption and diffusion behavior. This is also seen in Fig. $2 \mathrm{~b}$ as the water flux is relatively stable at low ethanol concentrations.

Fig. 4 shows the separation factor $\alpha_{E / W}$ as well as the selectivity $\sigma_{E / W}$ as a function of the mass fraction of ethanol in the feed. The observed separation factors of this study are in the same range as have been reported in literature [8,9,25,27,44,45]. 


\section{A)}

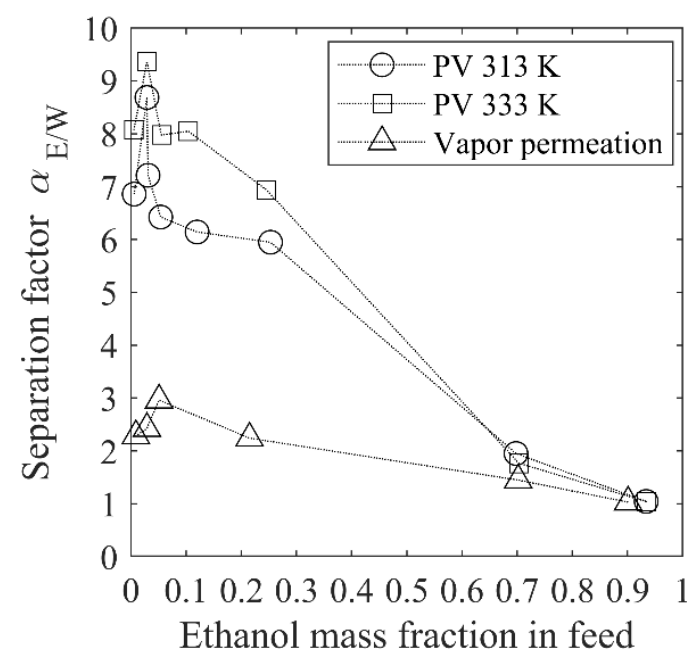

B)

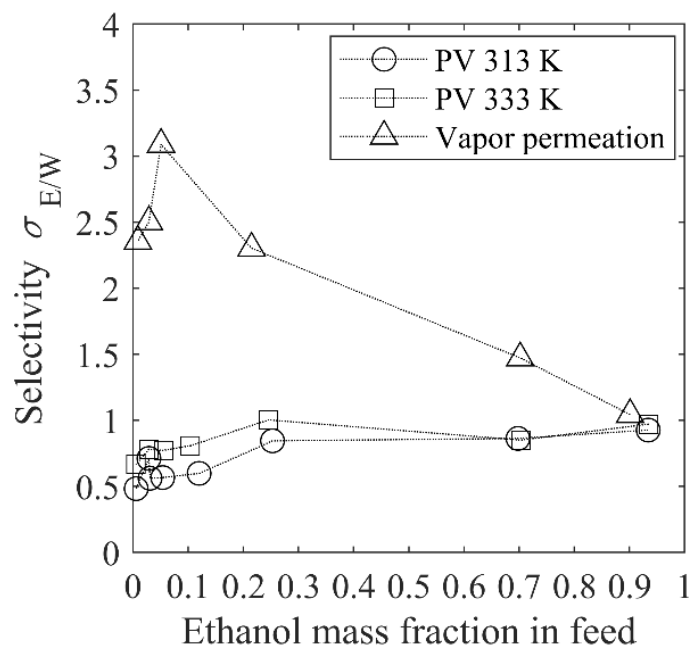

Figure 4. a) Separation factor and b) selectivity as a function of feed ethanol mass fraction in pervaporation experiments at $313 \mathrm{~K}(\circ)$ and $333 \mathrm{~K}(\square)$, and in vapor permeation experiments $(\Delta)$. The lines are guidance for the eye.

Fig. 4a, shows that the separation factor decreases with increasing ethanol concentration. At low ethanol concentration the separation factor exhibits variation, showing a peak at range 3-5 wt.\% feed ethanol concentration, in each dataset. The separation factor depends on both sorption and diffusion behavior but also on the driving force, particularly the fugacities of the component in the feed phase. The selectivity $\sigma_{i / j}$, on the other hand, includes the effect of the driving force as described in Eqs. (3)-(7) and is more directly related to the intrinsic properties of the membrane than separation factor. It can be seen in Fig. $4 \mathrm{~b}$ that the selectivity is quite stable and near unity in pervaporation conditions but decreases at low ethanol concentration range. In vapor permeation conditions the variation of selectivity is more distinct. In vapor permeation conditions, the vapor can be assumed to behave like an ideal gas mixture and the driving force can be described with partial pressure gradient, as described earlier in section 2.4. In contrast, in pervaporation conditions the driving force is the difference between the fugacity of a compound in the non-ideally behaving liquid feed and the ideally behaving gaseous 
permeate. If the separation performance depended only on the fugacity gradient over the membrane, the selectivity should remain constant. The changes in selectivity, i.e. the ratio of component permeances can be considered as an indication that there is significant interplay between the components during sorption or/and diffusion. The dependences of the s permeation of the components on the sorption and diffusion behavior is investigated in more detail next using a mathematical model.

\subsection{Modelling}

\subsubsection{Sorption}

Sorption of pure ethanol and water as well as their mixtures on PDMS were modeled using the extended Flory-Huggins model as described in Eqs. (8-(24). The penetrant-PDMS interaction parameter $\chi_{i m}$ was assumed to be dependent on both the volume fraction of PDMS and the temperature as shown in Eq. (10). The temperature dependence parameters for $\chi_{i \mathrm{~m}}$ were estimated based on data by Yang and Lue $[42,46]$. Both pure ethanol and water, and binary sorption data of Yang and Lue [42] performed at $298 \mathrm{~K}$ were used to estimate the PDMS volume fraction dependency for $\chi_{i m}$ as otherwise reaching credible description of the behavior would not have been possible. Instead, only the pure component sorption data from Yang and Lue [46] were used to estimate the temperature dependency parameters for $\chi_{i \mathrm{~m}}$. Furthermore, the interaction parameters of water and ethanol $\chi_{12}$ at different temperatures and compositions were estimated using the binary ethanol-water interaction behavior data from Aspen Plus data bank. The results and the used data are presented in the supplementary data.

The pure ethanol and water uptakes on PDMS at different temperatures both based on the model and experiments of Yang and Lue [42,46] are shown in Fig. 5 while the dependence on the ethanol feed concentration is presented in Fig. 6. The estimated parameters for the sorption model are presented in Table 1 . 
Table 1. Estimated penetrant-PDMS interaction temperature dependence parameter values.

\begin{tabular}{ccc} 
Parameter & Ethanol $(i=1)$ & Water $(i=2)$ \\
\hline$a_{i \mathrm{~m}, \mathrm{ref}}$ & 2.0992 & 4.5754 \\
$a_{i \mathrm{~m}, 1}$ & -0.0173 & 0.0974 \\
$b_{i \mathrm{~m}, \mathrm{ref}}$ & 0.0114 & $5.932 \cdot 10^{-4}$ \\
$b_{i \mathrm{~m}, 1}$ & -0.05255 & -0.00103 \\
$c_{i \mathrm{~m}, \mathrm{ref}}$ & -0.9317 & -0.9800 \\
$c_{i \mathrm{~m}, 1}$ & $7.0630 \cdot 10^{-6}$ & $3.0870 \cdot 10^{-3}$ \\
\hline
\end{tabular}

A)

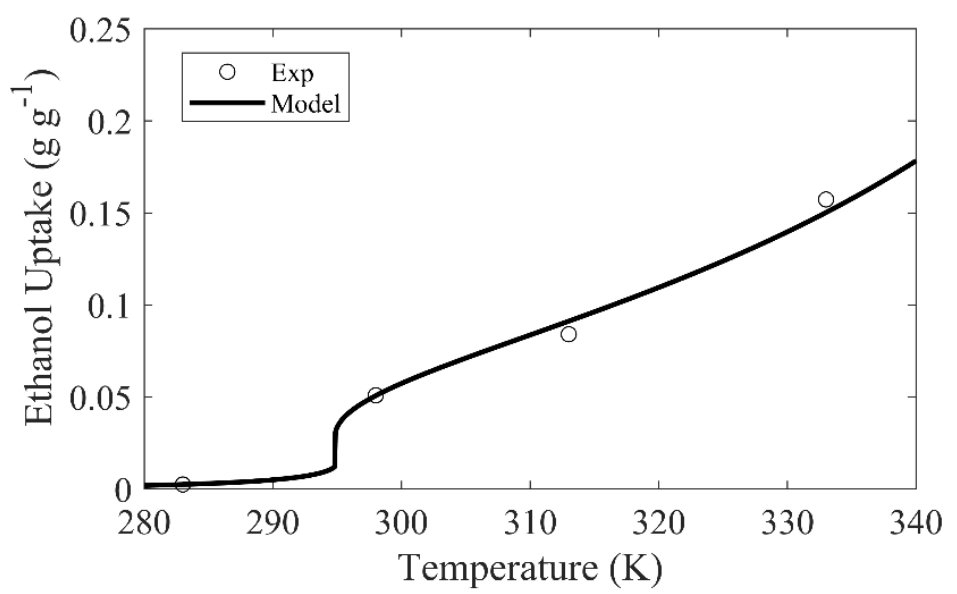

B)

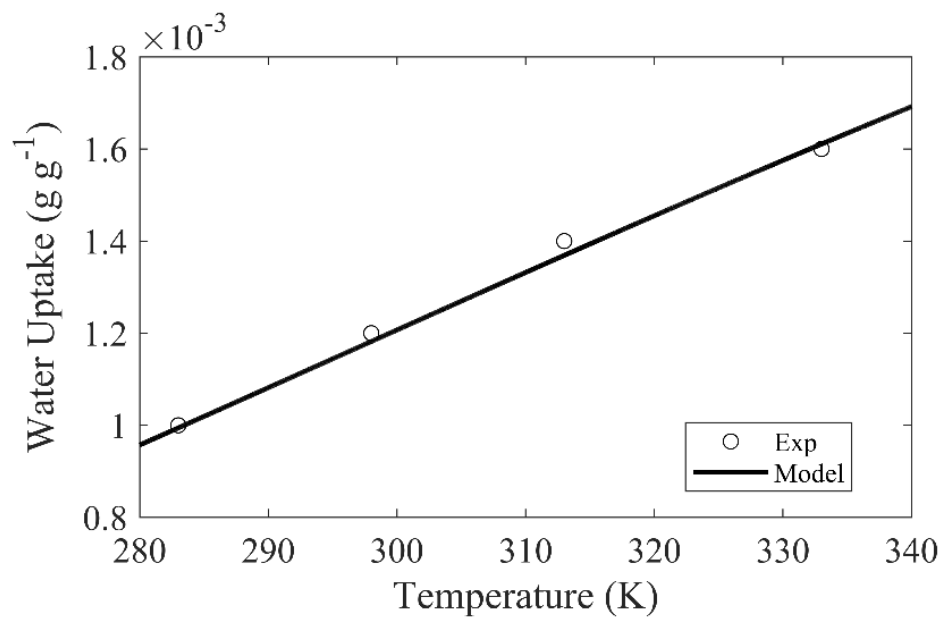

Figure 5. Pure a) ethanol and b) water uptakes on PDMS at different temperatures based on the experimental data of Yang and Lue [46] and the Flory-Huggins model of this study.

As can be seen in Fig. 5, the model describes well both the observed sorption behavior of pure ethanol and water at different temperatures. Sorption of water on PDMS behaves nearly linearly as a function of temperature while for ethanol there is clear non-linearity. As a result, the description of water sorption demanded simpler temperature dependence than ethanol.

However, from Fig. 5a it can be observed that the model predicts that the ethanol uptake changes considerably at around $296 \mathrm{~K}$. This observation cannot be verified based on the 
experimental sorption data of ethanol. Nevertheless, the model is used in this study at temperatures above $296 \mathrm{~K}$ and thus the uncertainties related to the sharp turn in ethanol uptake at lower temperatures does not have that high significance in the obtained results. More detailed description of the ethanol uptake model is found in supplementary material.
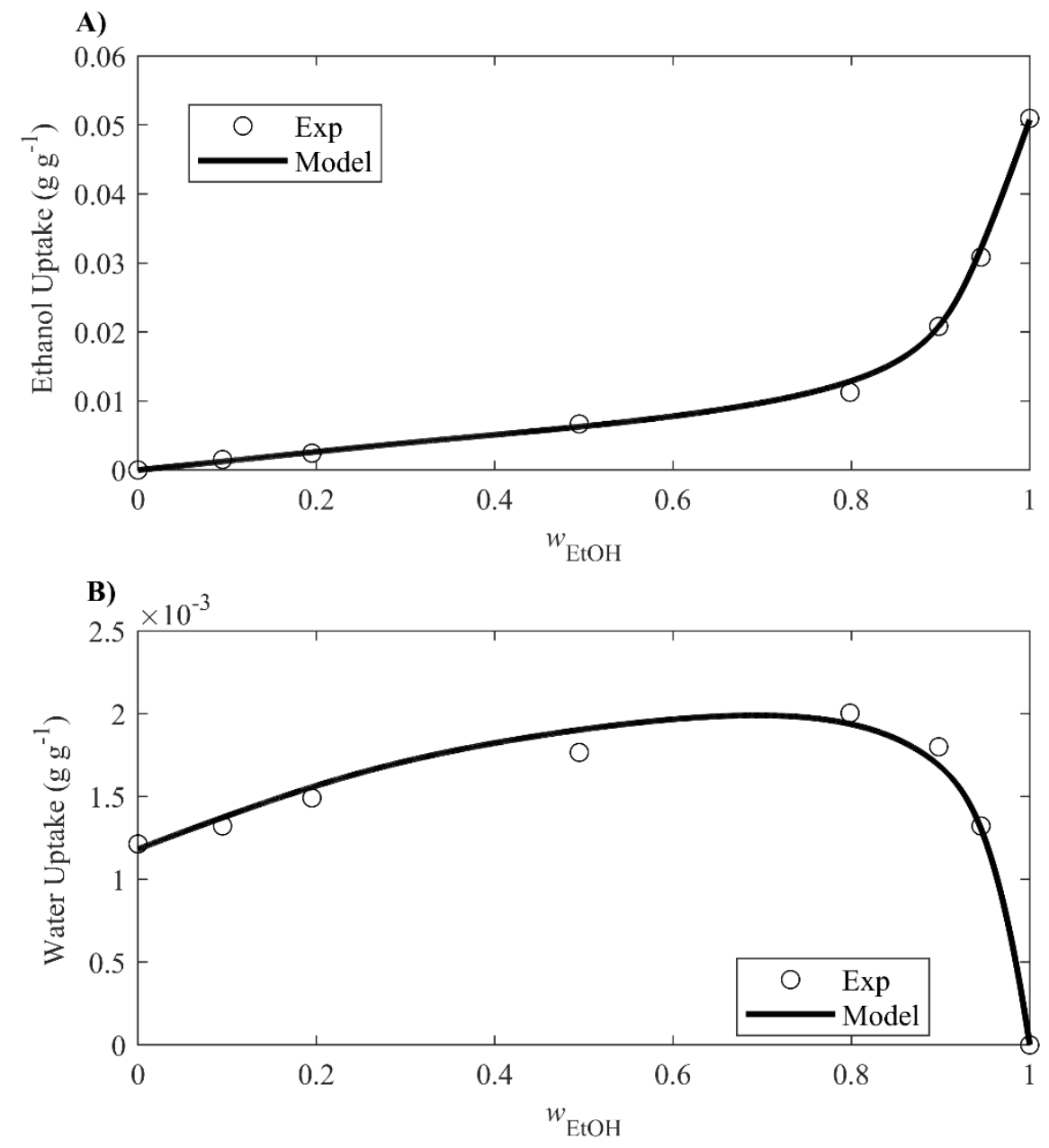

Figure 6. Sorbed amounts of a) ethanol and b) water as a function of the liquid ethanol mass fraction based on the sorption model (solid line) of this study and the experimental data of Yang and Lue [42] at $298 \mathrm{~K}(0)$.

As can be seen in Fig. 6, the model describes well the behavior of water and ethanol uptake at different feed concentrations. According to the model, the water uptake increases with increasing ethanol feed concentration and exhibits a maximum at around $70 \mathrm{wt} . \%$ of ethanol while the ethanol uptake increases monotonically as its feed concentration increases. These observations are in line with the experimental data. Comparison of Fig. 6 with Fig. 2 shows clear correlation between the flux and the sorption as a function of ethanol feed mass fraction. Furthermore, according to the model, the ethanol uptake is significantly higher than the uptake of water at the majority of the feed concentration range. Only at dilute ethanol concentration $(<10 \mathrm{wt} . \%)$ the sorption of the two components is at comparable level $(0.001 \mathrm{~g} / \mathrm{g}$, at $298 \mathrm{~K})$, or the water uptake even exceeds the one of ethanol.

With the described model, the uptake of ethanol and water in the experimental conditions were calculated. Due to the experimental setup, having turbulent flow region and membrane placed in thermostated oven, negligible concentration polarization at the feed side was assumed, and 
temperature gradient across the membrane was regarded insignificant. The effect of the support layers to the mass transport was taken into account at the permeate side as described in detail in the supplementary data.

\subsubsection{Diffusivity}

The values of the diffusion coefficients at infinite dilution $D_{i 0}$ were estimated separately for each experiment temperature based on the experimentally observed pure component fluxes using Fick's law, Eq. (25), and Eq. (27) for diffusion coefficient. The values of the pure component diffusivities were estimated from the pervaporation experiments performed at temperatures $313,333 \mathrm{~K}$ and vapor permeation experiments of ethanol at $362 \mathrm{~K}$ and water at $380 \mathrm{~K}$. The diffusivity values at other temperatures were approximated with linearization based on the experimental points. The values of the PDMS density and plasticization coefficients were obtained from literature [15]. Values of the plasticization coefficient were [15]: $\varepsilon_{11}=$ $-47.6, \varepsilon_{12}=-1.6, \varepsilon_{21}=-62.5$, and $\varepsilon_{22}=14$. Negative plasticization coefficients are associated with cases where diffusion coefficient decreases with increasing local concentration - behavior observed with polar solvents in apolar polymeric matrices [16]. The PDMS layer was assumed to have density of $1090 \mathrm{~kg} \mathrm{~m}^{-3}$ [15]. The obtained values of $D_{i 0}$ are shown in Table 2.

Table 2. Diffusion coefficients at different temperatures in pervaporation and vapor permeation experiments.

\begin{tabular}{lccll}
\hline Temperature & \multicolumn{2}{c}{$\begin{array}{c}\text { Diffusion coefficient } \\
D_{i 0} \times 10^{10} \mathrm{~m}^{2} \mathrm{~s}^{-1}\end{array}$} & Reference & Source of coefficients \\
\cline { 2 - 3 } & Ethanol & Water & & \\
\hline $313 \mathrm{~K}$ & 1.97 & 2.32 & this work & Pervaporation experiments \\
$333 \mathrm{~K}$ & 3.63 & 3.94 & this work & Pervaporation experiments \\
$362 \mathrm{~K}$ & 5.4 & & this work & Vapor permeation experiments \\
$380 \mathrm{~K}$ & & 13.3 & this work & Vapor permeation experiments \\
$313 \mathrm{~K}$ & 1.29 & 8.8 & {$[27]$} & Pervaporation experiments \\
$333 \mathrm{~K}$ & 2.08 & 12 & {$[27]$} & Pervaporation experiments \\
\hline
\end{tabular}

As can be seen in Table 2, $D_{i 0}$ of water is higher than the one of ethanol. This observation is reasoned by the smaller molecular size of water and is in line with the values of the literature. On the other hand, the values of $D_{i 0}$ increase as a function of the temperature as expected. The main difference to the values of Nasiri \& Aroujalian [27] is that the diffusivities obtained in this work are closer to each other, mainly because of water diffusivity obtained in this work is some degree smaller than the value they obtained. However, the differences can be explained with the differences in the sorption uptakes, experimental equipment operational differences and the PDMS structural characteristics.

To enable further evaluation and comparison of the diffusivities of this work with the literature studies, the average diffusion coefficients across the membrane were calculated for pure components using Eqs. (33)-(36). The calculated average diffusion coefficients at the experimental conditions of this work are presented in Table 3. 
Table 3. Average diffusion coefficients in the PDMS.

\begin{tabular}{|c|c|c|c|c|}
\hline \multirow[t]{2}{*}{ Temperature } & \multicolumn{2}{|c|}{$\begin{array}{l}\text { Diffusion coefficient } \\
\overline{D_{i}} \times 10^{10} \mathrm{~m}^{2} \mathrm{~s}^{-1}\end{array}$} & \multirow[t]{2}{*}{ Reference } & \multirow[t]{2}{*}{ Source of coefficients } \\
\hline & Ethanol & Water & & \\
\hline $313 \mathrm{~K}$ & 0.4 & 2.3 & this work & Pervaporation experiments \\
\hline $333 \mathrm{~K}$ & 0.5 & 3.8 & this work & Pervaporation experiments \\
\hline $362 \mathrm{~K}$ & 0.8 & & this work & Vapor permeation experiments \\
\hline $380 \mathrm{~K}$ & & 13.2 & this work & Vapor permeation experiments \\
\hline $298 \mathrm{~K}$ & 0.88 & 14.8 & {$[47]$} & Solvent uptake experiments \\
\hline $298 \mathrm{~K}$ & 0.22 & 0.4 & [48] & Solvent uptake experiments \\
\hline $298 \mathrm{~K}$ & 4.5 & 14.5 & [49] & Transient permeation experiments \\
\hline $298 \mathrm{~K}$ & 4.47 & 9.06 & {$[50]$} & Molecular simulation $^{\mathrm{a}}$ \\
\hline $298 \mathrm{~K}$ & 3.58 & 4.62 & {$[50]$} & Molecular simulation $^{b}$ \\
\hline $298 \mathrm{~K}$ & 4.73 & 20.6 & [27] & Molecular simulation \\
\hline $300 \mathrm{~K}$ & 4.4 & 13 & {$[51]$} & Molecular simulation \\
\hline $300 \mathrm{~K}$ & 2 & 15.3 & {$[52]$} & Molecular simulation \\
\hline $300 \mathrm{~K}$ & 0.05 & 0.25 & {$[52]$} & Molecular simulation $^{c}$ \\
\hline
\end{tabular}

\footnotetext{
${ }^{a}$ Membrane swelled with 10 wt.\% ethanol solution, ${ }^{b}$ Membrane swelled with 90 wt.\% ethanol

c Aggregated molecules
}

As can be seen in Table 3, the average diffusion coefficients of this work increase with temperature. It is noteworthy that the average diffusion coefficients and infinite dilution diffusion coefficients (Table 2) are almost equal for water, while for ethanol the average diffusion coefficient is significantly lower than the infinite dilution coefficient. Since the diffusion coefficients depend on the mass fractions according to Eq. (27), the different behaviors can be deduced to be based on the sorption behavior of the components. The water sorption uptake on PDMS is at low level regardless of the experimental conditions. Hence, due to the low sorption level of water, water diffuses at a comparable rate to the rate of diffusion it would have at infinite dilution conditions. Instead, sorption of ethanol is relatively high regardless of the temperature (see Fig. 5), and the plasticization effect on the PDMS matrix caused by the adsorbed ethanol is large. Thus, the average diffusion coefficient of ethanol is significantly lower than its diffusion coefficient at infinite dilution.

Furthermore, as can be seen in Table 3, the diffusion coefficients presented in the literature are typically one decade larger for water than for ethanol. Typical values reported in the literature at room temperature are approximately $2 \cdot 10^{-9}$ and $4 \cdot 10^{-10} \mathrm{~m}^{2} \mathrm{~s}^{-1}$ for water and ethanol, respectively [27,47-52]. However, as can be seen in Table 3, the differences between the literature data sources are high. In general, the diffusion in a polymer depends strongly on the type of the polymer, the number of molecules adsorbed on the polymer, and the size of the molecules, and whether they are free or aggregated. The comparison of the diffusion coefficients of this work for ethanol and water with the literature data is difficult as the coefficients of this work are based on higher temperature experimental data than the literature data. The formed Flory-Huggins model for sorption equilibrium shows strong temperature dependence for the uptake (see Fig. 5), resulting in high ethanol and water mass fractions in the membrane at temperatures higher than the room temperature. The plasticization caused by the high amount of adsorbed ethanol in turn decreases the rate of diffusion, and thus the observed diffusion coefficient. Without the plasticization effect, even steeper increase in the rate of 
diffusion would be observed with the increasing temperature and simultaneously increasing sorption gradient.

\subsubsection{Pervaporation and vapor permeation modelling}

The diffusional couplings between the penetrants were analyzed using the approximate Maxwell-Stefan equations, Eqs. (30)-(31), to describe the permeation behavior at different experimental conditions. In the model, the value of the diffusional interactions between the penetrants describing coefficient $D_{12}$ was evaluated for each temperature separately. However, the evaluation showed that the as long as the value is sufficiently low, it has not significant effect on the fluxes (See Supplementary material). The average diffusion coefficients $\bar{D}_{i, \mathrm{~m}}$ used in the model were defined by Eqs. (33)-(34) when the ethanol adsorption exceeded water adsorption and Eqs. (35)-(36) for the range where water adsorption was higher than the ethanol adsorption, i.e. at low ethanol feed concentration. The average diffusion coefficients of both components were observed to decrease with increasing ethanol concentration. The fluxes calculated with the Maxwell-Stefan model using the average diffusion coefficients are presented in Fig. 7. The model was applied with constant negative plasticization coefficients obtained from [15]. 
A)

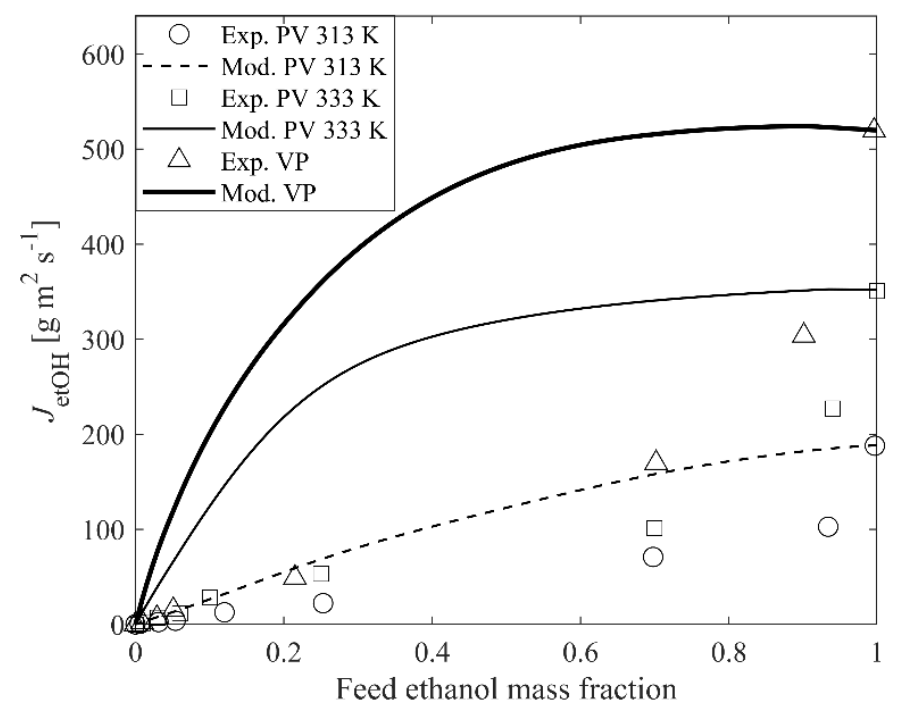

B)

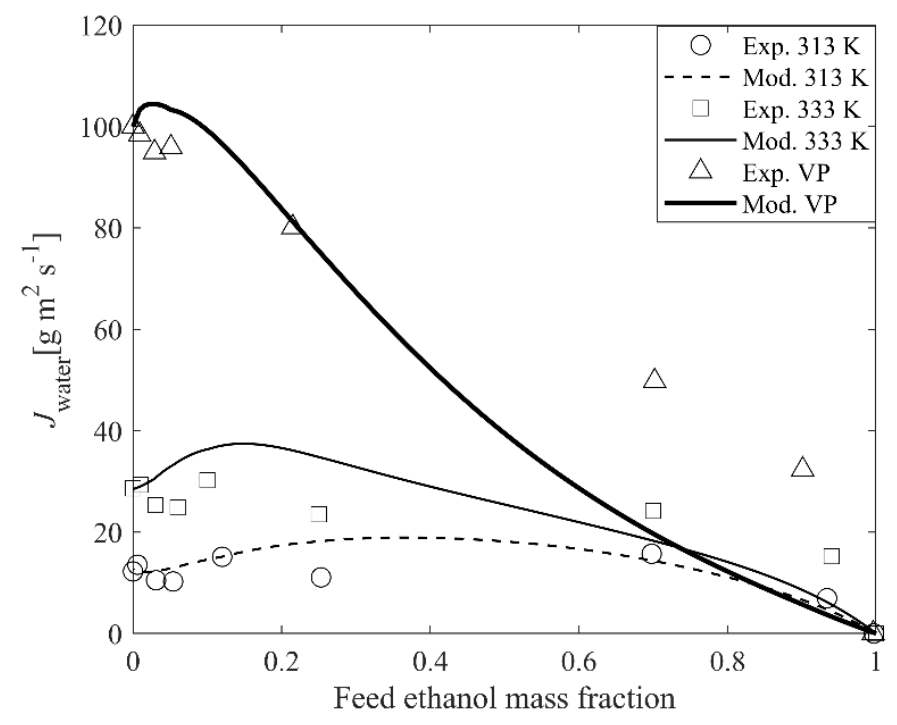

Figure 7. Experimentally observed fluxes (Exp.) and fluxes calculated with the model (Mod.) for a) ethanol and b) water as a function of feed ethanol mass fraction. $D_{12}=2.7 \cdot 10^{-14}$ at PV $313 \mathrm{~K}, D_{12}=4.1 \cdot 10^{-14}$ at PV $333 \mathrm{~K}$ and $D_{12}=2.0 \cdot 10^{-7}$ at vapor permeation conditions.

As can be seen in Fig. 7a, the model predicts higher ethanol fluxes in mixture permeation conditions than observed experimentally. In contrast, the fit of the model to the experimental water fluxes is relatively good but depends highly on the experimental conditions. The adjustable parameter, $D_{12}$, describing the reciprocal of mutual friction was fitted from the data. The parameter got value of $2.7 \cdot 10^{-14}$ at $313 \mathrm{~K}$ and $4.0 \cdot 10^{-14} \mathrm{~m}^{2} \mathrm{~s}^{-1}$ at $333 \mathrm{~K}$ which are significantly smaller than the values of $D_{i, \mathrm{~m}}$ for either of the penetrants in pervaporation condition. For comparison, the diffusion coefficients for the slower component ethanol were $4.3 \cdot 10^{-11} \mathrm{~m}^{2} \mathrm{~s}^{-1}$ at $313 \mathrm{~K}$ and $4.8 \cdot 10^{-11} \mathrm{~m}^{2} \mathrm{~s}^{-1}$ at $333 \mathrm{~K}$. The parameter $D_{12}$ represents the reciprocal of mutual friction, and the very small value indicates strong interaction between the penetrants. This parameter got larger value of $2.0 \cdot 10^{-7} \mathrm{~m}^{2} \mathrm{~s}^{-1}$ in vapor permeation conditions, indicating lower mutual friction effect in these conditions compared to lower temperatures and 
liquid phase feed. The prediction underestimates the water flux at high ethanol concentration in vapor permeation, despite the large value of binary exchange coefficient $D_{12}$. This may indicate that the used model overestimates the effect that plasticization caused by the penetrants has on the water flux. It is noteworthy that the model can predict the experimentally observed maximum of water flux at low ethanol concentration.

The estimated value of $D_{12}$ is very small in the pervaporation conditions. The fit of the model at the conditions is relatively good for water while for ethanol, the flux is overestimated. The coefficient $D_{12}$ can describe the slowing down effect of faster diffusing penetrant due to the retarding effect of the slower diffusing penetrant. In this case, ethanol is the slower of the penetrants based on the comparison of the diffusion coefficients represented in Tables 2 and 3 . Thus, only water can be slowed down through the effect of $D_{12}$ while the ethanol flux is overestimated regardless of the value of $D_{12}$. This implies that there exists a mutual slowing down effect, which causes also ethanol to permeate slower, but this effect cannot be described with the current model. However, in the model there are still uncertainties regarding the description of sorption and swelling of the membrane at different conditions, which could explain at least partially the observed behavior.

The mutual slowing down phenomenon has been denoted also by other authors $[16,27,32]$ and molecular simulations have revealed that the reason behind mutual slowing down might lie in molecular clustering between ethanol and water molecules in PDMS matrix due to hydrogen bonding. These clusters of multiple molecules diffuse significantly slower than a single molecule of either kind and represents itself as apparent decrease in diffusion rates of both individual penetrants. Krishna and van Baten [32] found strong hydrogen bonding tendency in diffusion of ethanol and water in zeolite. It was associated with binary exchange coefficient $D_{12}$ that was lower than the self-exchange coefficients, implying strong correlation effects in binary diffusion of ethanol and water. This is what is also seen in the results reported here. Also, the degree of clustering is lowered as the temperature increases [32], which may explain the difference between the vapor permeation and pervaporation results. It is reasonable to assume that ethanol and water molecules interact by forming hydrogen bonds also in PDMS, resulting in slower diffusion of both components, which is also suggested by Nasiri and Aroujalian [27] with molecular simulation studies with water and ethanol in PDMS.

In Fig. 7 the pervaporation and vapor permeation behavior as function of concentration is shown, and in Fig. 8 the role of temperature is investigated. As shown in Fig. 8a, using 3 wt.\% ethanol feed solution, the experimental fluxes of both ethanol and water increase with increasing temperature while the feed is in liquid phase (pervaporation). The transition of feed solution to vapor phase results in the decrease of the ethanol flux, while water flux continues to increase. This trend is easily explainable by the corresponding fugacity gradients, as seen in Fig. 8b. Due to the non-ideality of the feed mixture, the activity coefficient of ethanol in liquid is high, and its fugacity is higher than its relative proportion, $3 \mathrm{wt} . \%$, in the solution, while in vapor phase the fugacity is more directly dependent on the component concentration and partial pressure. Even though the fugacity gradient is an easy way to get an idea of the trends of the fluxes it is not enough to predict the fluxes. 
A)
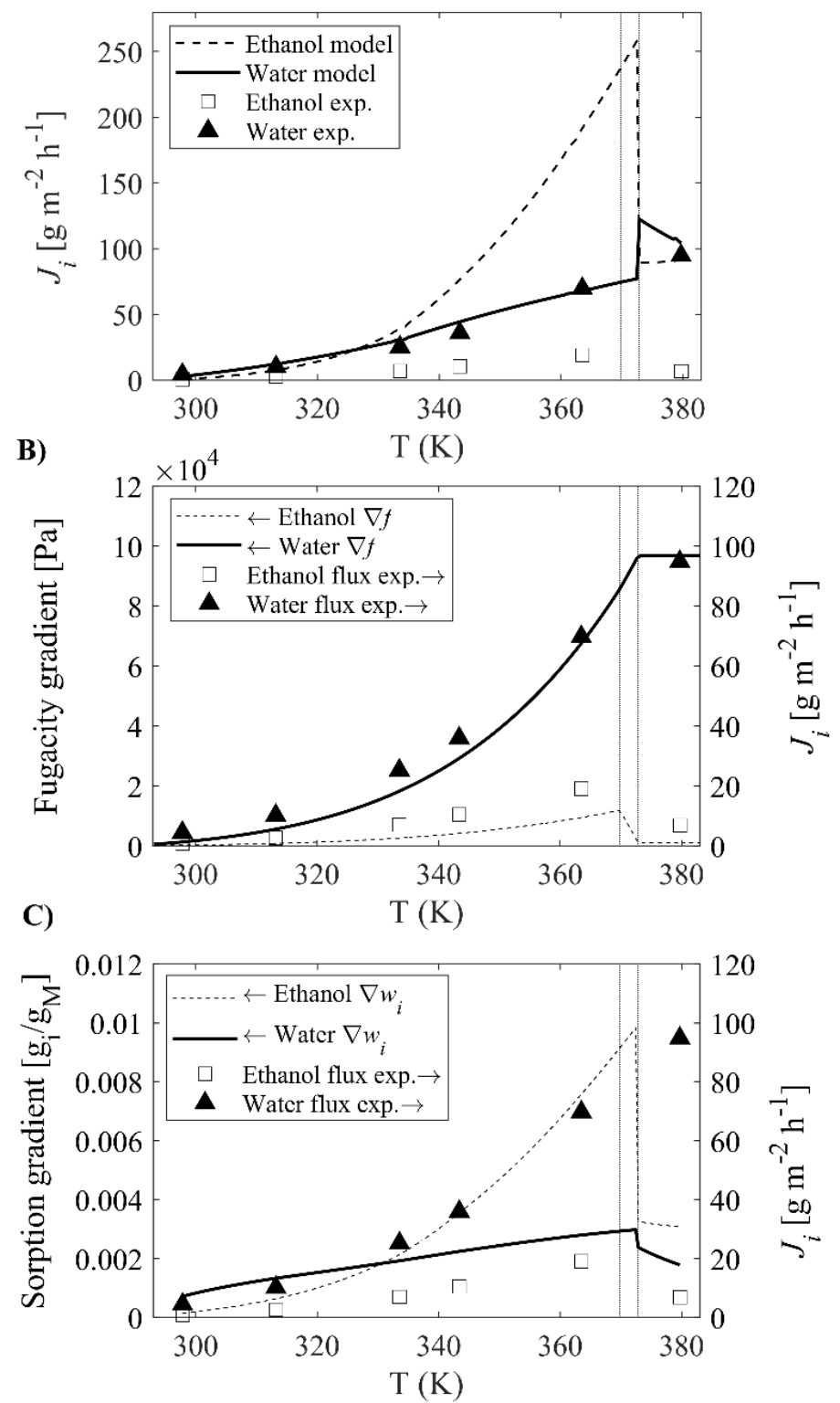

Figure 8. a) Experimental and modelled ethanol and water fluxes, b) fugacity gradients of both components, and c) sorption gradient of ethanol and water at different temperatures. In b) and c) the experimental fluxes are on the right y-axis helping interpretation. The feed composition is $3 \mathrm{wt} . \%$ ethanol. The vertical lines show the boiling and dew point temperatures of the feed mixture at $P=1.013$ bar. For mixed phase range, model for liquid phase is used. $D_{12}$ is based on the values of PV and VP isotherms.

The fluxes predicted with Maxwell-Stefan model overestimate the flux of ethanol but give relatively good fit for water flux. (Fig. 8a). The binary exchange coefficient at each temperature is based on the values estimated for the isotherms presented in Fig. 7. According to the modified Flory-Huggins model, Eqs. (8)-(9), the ethanol uptake steeply increases with increasing temperature, creating also a larger sorption gradient as shown in Fig. 8c. The uptake from vapor phase is less than from liquid phase. In addition to the increased uptake, also the diffusion of the molecules should increase with the increasing temperature. Therefore, theoretically the fluxes are expected to be high at higher temperatures. However, as seen in Fig. 8a, the 
experimental ethanol fluxes do not meet the expectations of high flux at high temperature, indicating that there are phenomena that hinder the flux of the ethanol. The binary exchange coefficient $D_{12}$ can be used to approximate the slowdown of the faster water molecule, but the model as such is not capable of predicting that also the slower ethanol molecule would diffuse slower in presence of a large amount of water. The difference between the expected and the observed ethanol flux is large, indicating severe coupling effects. As a conclusion, the prediction of pervaporation and vapor permeation fluxes of binary water and ethanol mixtures is difficult due to the strong interactions between the components. There remains need for further research on how to estimate the binary effects in a reliable way.

\section{Conclusions}

The behavior of a supported PDMS membrane in ethanol-water separation was investigated using experimental and modelling work. The experimental work included comprehensive coverage of pure component permeation and wide range of binary ethanol-water mixture separation experiments at pervaporation and vapor permeation conditions. As a whole, the observed fluxes correlate with the changes of the component fugacities and the sorption gradients. However, the interactions between the penetrants have a significant role in determining the separation performance. The interactions between the penetrants were analyzed with the help of a formed Maxwell-Stefan model.

According to the formed model, both water and ethanol permeation are slowed down from the permeation rates observed with pure feeds. The observation is significant as it is theoretically justified that the slower compound, i.e. ethanol, slows the diffusion of the faster diffusing compound, i.e. water, but not that both were slowed down. The observed ethanol flux in mixture permeation was lower than what was expected based on the formed Maxwell-Stefan model. This indicates that the interactions between the penetrants are high and a likely reason for this is the hydrogen bonding between the penetrants, which may result in the formation of molecular clusters between ethanol and water molecules. The significance of molecular clustering in the water-ethanol separation cannot be verified with the used model as there are uncertainties present in the applied sorption model and data. Thus, further sorption equilibrium experiments are needed in the future work. Furthermore, the model should be developed further by including a theoretically credible description of the potentially occurring molecular clustering.

\section{Acknowledgements}

The experimental work was done as a part of the 'Sustainable production concepts on integrated biorefining industry' project (decision number 286633) financed by the Academy of Finland. The work was supported by grants from Emil Aaltonen Foundation and Tauno Tönning Foundation. The help received from Center of Microscopy and Nanotechnology, University of Oulu in membrane sample preparation and microscopic analysis is highly appreciated. 


\section{Nomenclature}

$a$

A

$b$

C

$d$

e

D

$f$

$J$

$p$

$P$

$t$

$T$

$u$

$\bar{V}$

w

$x$

$y$

$z$

\section{Greek letters}

$\alpha$

$\gamma$

$\varepsilon$

$\Pi$

$\rho$

$\sigma$

$\varphi$

$\chi$

\section{Superscript}

temperature dependent interaction parameter in Flory-Huggins model or activity of a compound

membrane area $\left[\mathrm{m}^{2}\right]$

temperature dependent interaction parameter in Flory-Huggins model

temperature dependent interaction parameter in Flory-Huggins model

temperature dependent interaction parameter in Flory-Huggins model

temperature dependent interaction parameter in Flory-Huggins model

diffusion coefficient

fugacity $[\mathrm{Pa}]$

flux $\left[\mathrm{kg} \mathrm{m}^{-2} \mathrm{~h}^{-1}\right]$ or $\left[\mathrm{mol} \mathrm{m}^{-2} \mathrm{~h}^{-1}\right]$

partial pressure $[\mathrm{Pa}]$

pressure $[\mathrm{Pa}]$

time $[\mathrm{h}]$ or $[\mathrm{s}]$

temperature $[\mathrm{K}]$ or $\left[{ }^{\circ} \mathrm{C}\right]$

volume fraction in bulk fluid phase [-]

partial molar volume $\left[\mathrm{m}^{3} \mathrm{~mol}^{-1}\right]$

mass fraction [-]

mole fraction on feed side or in liquid phase [-]

mole fraction on permeate side or in vapor phase [-]

membrane thickness [m]

separation factor [-]

activity coefficient [-]

plasticization coefficient [-]

permeance $\left[\mathrm{mol} \mathrm{m}^{-2} \mathrm{~h}^{-1} \mathrm{~Pa}^{-1}\right.$ ]

density $\left[\mathrm{kg} \mathrm{m}^{-3}\right]$

selectivity [-]

volume fraction [-]

Flory-Huggins interaction parameter [-] 


\section{Subscript}

0

avg

bulk

E,etOH,1

feed, F

$i$

$j$

$i / j$

$\mathrm{m}$

perm, $\mathrm{P}$

ref

W,water, 2 zero concentration (diffusion coefficient)

average

bulk phase

ethanol

feed side

component $i$

component $j$

separation of component $i$ from component $j$

PDMS, polymer membrane matrix

permeate side

reference

water 


\section{References}

[1] F. Lipnizki, R.W. Field, P.-K. Ten, Pervaporation-based hybrid process: a review of process design, applications and economics, J. Memb. Sci. 153 (1999) 183-210. doi:10.1016/S0376-7388(98)00253-1.

[2] Q. Kang, B. Van Der Bruggen, R. Dewil, J. Baeyens, T. Tan, Hybrid operation of the bio-ethanol fermentation, Sep. Purif. Technol. 149 (2015) 322-330. doi:10.1016/j.seppur.2015.05.007.

[3] B. Van der Bruggen, P. Luis, Pervaporation, in: Prog. Filtr. Sep., Academic Press, 2015: pp. 101-154. doi:10.1016/B978-0-12-384746-1.00004-5.

[4] G.R. Harvianto, F. Ahmad, L.C. Nhien, M. Lee, Vapor permeation-distillation hybrid processes for cost-effective isopropanol dehydration: modeling, simulation and optimization, J. Memb. Sci. 497 (2016) 108-119. doi:10.1016/J.MEMSCI.2015.09.032.

[5] A. Singh, G.P. Rangaiah, Development and optimization of a novel process of doubleeffect distillation with vapor recompression for bioethanol recovery and vapor permeation for bioethanol dehydration, J. Chem. Technol. Biotechnol. 94 (2019) 10411056. doi:10.1002/jctb.5851.

[6] C. Wang, Z. Zhang, X. Zhang, J. Gao, B. Stewart, Energy-saving hybrid processes combining pressure-swing reactive distillation and pervaporation membrane for n-propyl acetate production, Sep. Purif. Technol. 221 (2019) 1-11. doi:10.1016/J.SEPPUR.2019.03.074.

[7] A. Tgarguifa, S. Abderafi, T. Bounahmidi, Energy efficiency improvement of a bioethanol distillery, by replacing a rectifying column with a pervaporation unit, Renew. Energy. 122 (2018) 239-250. doi:10.1016/J.RENENE.2018.01.112.

[8] C. Fu, D. Cai, S. Hu, Q. Miao, Y. Wang, P. Qin, Z. Wang, T. Tan, Ethanol fermentation integrated with PDMS composite membrane: An effective process, Bioresour. Technol. 200 (2016) 648-657. doi://dx.doi.org/10.1016/j.biortech.2015.09.117.

[9] W. Sun, W. Jia, C. Xia, W. Zhang, Z. Ren, Study of in situ ethanol recovery via vapor permeation from fermentation, J. Memb. Sci. 530 (2017) 192-200. doi://doi.org/10.1016/j.memsci.2017.02.034.

[10] S. Fan, Z. Xiao, M. Li, S. Li, T. Zhou, Y. Hu, S. Wu, Pervaporation performance in PDMS membrane bioreactor for ethanol recovery with running water and air as coolants at room temperature, J. Chem. Technol. Biotechnol. 92 (2017) 292-297. doi:10.1002/jctb.5004.

[11] G. Liu, W. Wei, H. Wu, X. Dong, M. Jiang, W. Jin, Pervaporation performance of PDMS/ceramic composite membrane in acetone butanol ethanol (ABE) fermentationPV coupled process, J. Memb. Sci. 373 (2011) 121-129. doi://dx.doi.org/10.1016/j.memsci.2011.02.042.

[12] G. Liu, L. Gan, S. Liu, H. Zhou, W. Wei, W. Jin, PDMS/ceramic composite membrane for pervaporation separation of acetone-butanol-ethanol (ABE) aqueous solutions and its application in intensification of ABE fermentation process, Chem. Eng. Process. Process Intensif. 86 (2014) 162-172. doi://dx.doi.org/10.1016/j.cep.2014.06.013.

[13] D. Cai, S. Hu, Q. Miao, C. Chen, H. Chen, C. Zhang, P. Li, P. Qin, T. Tan, Two-stage 
pervaporation process for effective in situ removal acetone-butanol-ethanol from fermentation broth, Bioresour. Technol. $224 \quad$ (2017) 380-388. doi://doi.org/10.1016/j.biortech.2016.11.010.

[14] P. Zheng, C. Li, N. Wang, J. Li, Q. An, The potential of pervaporation for biofuel recovery from fermentation: An energy consumption point of view, Chinese J. Chem. Eng. (2018). doi://doi.org/10.1016/j.cjche.2018.09.025.

[15] T.-H. Yang, S.J. Lue, Coupled concentration-dependent diffusivities of ethanol/water mixtures through a polymeric membrane: Effect on pervaporative flux and diffusivity profiles, J. Memb. Sci. 443 (2013) 1-9. doi://dx.doi.org/10.1016/j.memsci.2013.05.002.

[16] E. Favre, P. Schaetzel, Q.T. Nguygen, R. Clément, J. Néel, Sorption, diffusion and vapor permeation of various penetrants through dense poly(dimethylsiloxane) membranes: a transport analysis, J. Memb. Sci. 92 (1994) 169-184. doi:10.1016/0376-7388(94)000603 .

[17] S. Li, F. Qin, P. Qin, M.N. Karim, T. Tan, Preparation of PDMS membrane using water as solvent for pervaporation separation of butanol-water mixture, Green Chem. 15 (2013) 2180-2190. doi:10.1039/c3gc40291f.

[18] F. Qin, S. Li, P. Qin, M.N. Karim, T. Tan, A PDMS membrane with high pervaporation performance for the separation of furfural and its potential in industrial application, Green Chem. 16 (2014) 1262-1273. doi:10.1039/c3gc41867g.

[19] L. Ji, B. Shi, L. Wang, Pervaporation separation of ethanol/water mixture using modified zeolite filled PDMS membranes, J. Appl. Polym. Sci. 132 (2015). doi:10.1002/app.41897.

[20] R.D. Offeman, C.N. Ludvik, A novel method to fabricate high permeance, high selectivity thin-film composite membranes, J. Memb. Sci. 380 (2011) 163-170. doi://doi.org/10.1016/j.memsci.2011.07.005.

[21] P. Peng, B. Shi, Y. Lan, A Review of Membrane Materials for Ethanol Recovery by

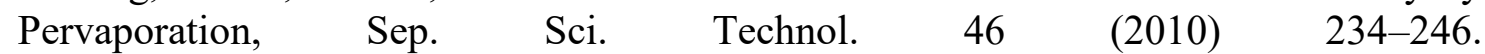
doi:10.1080/01496395.2010.504681.

[22] W. Wei, S. Xia, G. Liu, X. Dong, W. Jin, N. Xu, Effects of polydimethylsiloxane (PDMS) molecular weight on performance of PDMS/ceramic composite membranes, J. Memb. Sci. 375 (2011) 334-344. doi:10.1016/j.memsci.2011.03.059.

[23] Z. Dong, G. Liu, S. Liu, Z. Liu, W. Jin, High performance ceramic hollow fiber supported PDMS composite pervaporation membrane for bio-butanol recovery, J. Memb. Sci. 450 (2014) 38-47. doi:10.1016/j.memsci.2013.08.039.

[24] D. Korelskiy, T. Leppäjärvi, H. Zhou, M. Grahn, J. Tanskanen, J. Hedlund, High flux MFI membranes for pervaporation, J. Memb. Sci. 427 (2013) 381-389. doi://dx.doi.org/10.1016/j.memsci.2012.10.016.

[25] T. Mohammadi, A. Aroujalian, A. Bakhshi, Pervaporation of dilute alcoholic mixtures using PDMS membrane, Chem. Eng. Sci. 60 (2005) 1875-1880. doi://doi.org/10.1016/j.ces.2004.11.039.

[26] F. Xiangli, Y. Chen, W. Jin, N. Xu, Polydimethylsiloxane (PDMS)/Ceramic Composite Membrane with High Flux for Pervaporation of Ethanol-Water Mixtures, Ind. Eng. Chem. Res. 46 (2007) 2224-2230. doi:10.1021/ie0610290. 
[27] H. Nasiri, A. Aroujalian, A novel model based on cluster formation for pervaporation separation of polar components from aqueous solutions, Sep. Purif. Technol. 72 (2010) 13-21. doi://doi.org/10.1016/j.seppur.2009.12.020.

[28] P. Peng, B. Shi, Y. Lan, Preparation of PDMS — Silica Nanocomposite Membranes with Silane Coupling for Recovering Ethanol by Pervaporation, Sep. Sci. Technol. 46 (2011) 420-427. doi:10.1080/01496395.2010.527896.

[29] R. Krishna, Describing mixture permeation across polymeric membranes by a combination of Maxwell-Stefan and Flory-Huggins models, Polymer (Guildf). 103 (2016) 124-131. doi://dx.doi.org/10.1016/j.polymer.2016.09.051.

[30] P. Shao, R.Y.M. Huang, Polymeric membrane pervaporation, J. Memb. Sci. 287 (2007) 162-179. doi://doi.org/10.1016/j.memsci.2006.10.043.

[31] M.S. Schehlmann, E. Wiedemann, R.N. Lichtenthaler, Pervaporation and vapor permeation at the azeotropic point or in the vicinity of the LLE boundary phases of organic/aqueous mixtures, J. Memb. Sci. 107 (1995) 277-282. doi://dx.doi.org/10.1016/0376-7388(95)00142-6.

[32] R. Krishna, J.M. Van Baten, Hydrogen bonding effects in adsorption of water-alcohol mixtures in zeolites and the consequences for the characteristics of the Maxwell-Stefan diffusivities, Langmuir. 26 (2010) 10854-10867. doi:10.1021/1a100737c.

[33] P. Izák, L. Bartovská, K. Friess, M. Šípek, P. Uchytil, Description of binary liquid mixtures transport through non-porous membrane by modified Maxwell-Stefan equations, J. Memb. Sci. 214 (2003) 293-309. doi:10.1016/S0376-7388(02)00580-X.

[34] A. Ebneyamini, H. Azimi, J. Thibault, F.H. Tezel, Description of butanol aqueous solution transport through commercial PDMS pervaporation membrane using extended Maxwell-Stefan model, Sep. Sci. Technol. 53 (2018) 1611-1627. doi:10.1080/01496395.2018.1441303.

[35] T. Leppäjärvi, I. Malinen, D. Korelskiy, J. Hedlund, J. Tanskanen, Maxwell-Stefan Modeling of Ethanol and Water Unary Pervaporation through a High-Silica MFI Zeolite Membrane, Ind. Eng. Chem. Res. 53 (2014) 323-332. doi:10.1021/ie400814z.

[36] A. Heintz, W. Stephan, A generalized solution - diffusion model of the pervaporation process through composite membranes Part II. Concentration polarization, coupled diffusion and the influence of the porous support layer, J. Memb. Sci. 89 (1994) 153169. doi://doi.org/10.1016/0376-7388(93)E0223-7.

[37] J. Abraham, T. Jose, G. Moni, S.C. George, N. Kalarikkal, S. Thomas, Ionic liquid modified multiwalled carbon nanotube embedded styrene butadiene rubber membranes for the selective removal of toluene from toluene/methanol mixture via pervaporation, $\mathrm{J}$. $\begin{array}{llllll}\text { Taiwan } & \text { Inst. } & \text { Chem. } & \text { Eng. } & 95 & \text { (2019) }\end{array}$ doi:https://doi.org/10.1016/j.jtice.2018.09.019.

[38] M. Aghakarimiha, A. Raisi, G. Pazuki, A compositional model based on SAFT-VR and Maxwell-Stefan equations for pervaporative separation of aroma compounds from aqueous solutions, J. Mol. Liq. $250 \quad$ (2018) 212-222. doi:10.1016/J.MOLLIQ.2017.11.165.

[39] R.W. Baker, J.G. Wijmans, Y. Huang, Permeability, permeance and selectivity: A preferred way of reporting pervaporation performance data, J. Memb. Sci. 348 (2010) 
346-352. doi://dx.doi.org/10.1016/j.memsci.2009.11.022.

[40] M. Weyd, H. Richter, P. Puhlfürß, I. Voigt, C. Hamel, A. Seidel-Morgenstern, Transport of binary water-ethanol mixtures through a multilayer hydrophobic zeolite membrane, J. Memb. Sci. 307 (2008) 239-248. doi://dx.doi.org/10.1016/j.memsci.2007.09.032.

[41] F.T. de Bruijn, L. Sun, Ž. Olujić, P.J. Jansens, F. Kapteijn, Influence of the support layer on the flux limitation in pervaporation, J. Memb. Sci. 223 (2003) 141-156. doi://doi.org/10.1016/S0376-7388(03)00318-1.

[42] T.-H. Yang, S.J. Lue, Modeling sorption behavior for ethanol/water mixtures in a crosslinked polydimethylsiloxane membrane using the Flory-Huggins equation, J. Macromol. Sci. Part B Phys. 52 (2013) 1009-1029. doi:10.1080/00222348.2012.750992.

[43] R.B. Long, Liquid permeation through plastic films, Ind. Eng. Chem. Fundam. 4 (1965) 445-451. doi:10.1021/i160016a015.

[44] G. Liu, F. Xiangli, W. Wei, S. Liu, W. Jin, Improved performance of PDMS/ceramic composite pervaporation membranes by ZSM-5 homogeneously dispersed in PDMS via a surface graft/coating approach, Chem. Eng. J. 174 (2011) 495-503. doi://dx.doi.org/10.1016/j.cej.2011.06.004.

[45] A. Rozicka, J. Niemistö, R.L. Keiski, W. Kujawski, Apparent and intrinsic properties of commercial PDMS based membranes in pervaporative removal of acetone, butanol and ethanol from binary aqueous mixtures, J. Memb. Sci. 453 (2014) 108-118. doi:10.1016/j.memsci.2013.10.065.

[46] T.-H. Yang, S.J. Lue, UNIQUAC and UNIQUAC-HB models for the sorption behavior of ethanol/water mixtures in a cross-linked polydimethylsiloxane membrane, J. Memb. Sci. 415-416 (2012) 534-545. doi://doi.org/10.1016/j.memsci.2012.05.041.

[47] S.J. Lue, T.-H. Yang, K.-S. Chang, K.-L. Tung, Water diffusivity suppression and ethanol-over-water diffusion selectivity enhancement for ethanol/water mixtures in polydimethylsiloxane-zeolite membranes, J. Memb. Sci. 415-416 (2012) 635-643. doi://doi.org/10.1016/j.memsci.2012.05.044.

[48] S.J. Lue, C.-F. Chien, K.P.O. Mahesh, Pervaporative concentration of ethanol-water mixtures using heterogeneous polydimethylsiloxane (PDMS) mixed matrix membranes, J. Memb. Sci. 384 (2011) 17-26. doi:10.1016/J.MEMSCI.2011.08.060.

[49] K.-I. Okamoto, S. Nishioka, S. Tsuru, S. Sasaki, K. Tanaka, H. Kita, Sorption and Pervaporation of Water-Organic Liquid Mixtures through Polydimethylsiloxane, Kobunshi Ronbunshu. 45 (1988) 993-999. doi:10.1295/koron.45.993.

[50] K.-S. Chang, Y.-C. Chung, T.-H. Yang, S.J. Lue, K.-L. Tung, Y.-F. Lin, Free volume and alcohol transport properties of PDMS membranes: Insights of nano-structure and interfacial affinity from molecular modeling, J. Memb. Sci. 417-418 (2012) 119-130. doi://doi.org/10.1016/j.memsci.2012.06.019.

[51] L. Fritz, D. Hofmann, Molecular dynamics simulations of the transport of water-ethanol mixtures through polydimethylsiloxane membranes, Polymer (Guildf). 38 (1997) 10351045. doi:10.1016/S0032-3861(96)00600-3.

[52] Y. Tamai, H. Tanaka, K. Nakanishi, Molecular Simulation of Permeation of Small Penetrants through Membranes. 1. Diffusion Coefficients, Macromolecules. 27 (1994) 4498-4508. doi:10.1021/ma00094a011. 